\title{
The Role of Anharmonicity in Hydrogen-Bonded Systems: The Case of Water Clusters
}

\author{
Berhane Temelso and George C. Shields* \\ Dean's Office, College of Arts and Sciences, Department of Chemistry, Bucknell University, Lewisburg, Pennsylvania 17837, \\ United States
}

Supporting Information

ABSTRACT: The nature of vibrational anharmonicity has been examined for the case of small water clusters using second-order vibrational perturbation theory (VPT2) applied on second-order Møller-Plesset perturbation theory (MP2) potential energy surfaces. Using a training set of 16 water clusters $\left(\mathrm{H}_{2} \mathrm{O}\right)_{n=2-6,8,9}$ with a total of 723 vibrational modes, we determined scaling factors that map the harmonic frequencies onto anharmonic ones. The intermolecular modes were found to be substantially more anharmonic than intramolecular bending and stretching modes. Due to the varying levels of anharmonicity of the intermolecular and intramolecular modes, different frequency scaling factors for each region were necessary to achieve the highest accuracy. Furthermore, new scaling factors for zero-point vibrational energies (ZPVE) and vibrational corrections to the enthalpy $\left(\Delta H_{\text {vib }}\right)$ and the entropy $\left(S_{\mathrm{vib}}\right)$ have been determined. All the scaling factors reported in this study are different from previous works in that they are intended for hydrogen-bonded systems, while others were built using experimental frequencies of covalently bonded systems. An application of our scaling factors to the vibrational frequencies of water dimer and thermodynamic functions of 11 larger water clusters highlights the importance of anharmonic effects in hydrogen-bonded systems.

\section{INTRODUCTION}

It has long been recognized that comparison of calculated harmonic vibrational frequencies $(\omega)$ with observed fundamental frequencies $(v)$ requires an empirical correction to account for vibrational anharmonicity and inherent errors in the electronic structure calculations. ${ }^{1-12}$ There are three main reasons for scaling calculated harmonic vibrational frequencies to approximate experimental frequencies. First, scaling corrects for deviation from the harmonic oscillator model as a consequence of anharmonicity. The deviation could be mild in the case of highfrequency stretching modes where the vibrational potential around the equilibrium geometry of the molecule is deep and well-described by a harmonic oscillator potential. The difference between this harmonic potential and the more appropriate Morse potential is small, and a simple scaling factor goes a long way in reducing the disparity between the two. However, there are extreme cases of anharmonicity where the "vibrational" potential has multiple shallow minima and the motion is better described as an internal rotation, ring inversion, or a pseudorotation. ${ }^{13-17}$ This is most common in low-frequency modes and cannot be easily remedied by introducing scaling factors. In cases where the harmonic approximation works well, a second reason for using scaling factors is that they can correct for the incompleteness of the basis set and electron correlation treatment that is inherent in the most practical quantum mechanical calculations. Scaling factors allow one to perform vibrational frequency analysis using a modest basis set and electron correlation method and to improve the quality of the calculated frequencies by scaling with a prescribed multiplicative factor. A third source of error in harmonic vibrational frequencies is the coupling of different modes, but accounting for these requires the calculation of coupled (as opposed to independent normal mode) vibrational wave functions. In principle, methods like vibrational configuration interaction $(\mathrm{VCI})^{18-20}$ and the Huang-Braams-Bowman $(\mathrm{HBB})^{21}$ potential can give exact vibrational wave functions, but they are not practical for systems with more than 10 atoms.

Anharmonic correction for rigid and semirigid systems typically amounts to $3-5 \%$ of harmonic frequencies computed using second-order Møller-Plesset perturbation theory (MP2) and Becke, three-parameter, Lee-Yang-Parr (B3LYP) methods and about $10 \%$ using Hartree-Fock (HF) methods with modest basis sets. Errors in harmonic vibrational frequencies are systematic, and it is possible to determine scaling factors by comparing calculated frequencies with experimental ones. Most scaling factors reported in the literature make use of large databases of experimental vibrational frequencies to systematically improve harmonic frequencies. By least-squares fitting of harmonic frequencies to experimental frequencies, many scaling factors have been suggested for a host of methods and basis sets. Radom et al. ${ }^{4,7}$ have obtained scaling factors for vibrational frequencies, zero point vibrational energies (ZPVE) and vibrational enthalpies $\left(\Delta H_{\mathrm{vib}}\right)$ and entropies $\left(S_{\mathrm{vib}}\right)$. They suggested different scaling factors for high- and low-frequency modes, with the high modes being scaled by $0.95-0.97$ and the inverse of the low modes being scaled by $1.01-1.04$ for MP2 theory with aug-cc-pVNZ basis sets where $N=D-Q$. A similar work by Sinha et al..$^{5}$ suggests split scaling of fundamental modes and lowfrequency modes. The recommended fundamental and inverse lowfrequency scaling factors for MP2 theory were 0.9604 and 1.0999 for the aug-cc-pVDZ basis set, 0.9557 and 1.0634 for the aug-cc-pVTZ basis set, and 0.9601 and 1.0698 for the aug-cc-pVQZ basis set. Halls et al. $^{12}$ have determined frequency scaling factors for various

Received: May 15, 2011

Published: July 15, 2011 
methods along with the Sadlej pVTZ electric property basis set and found that dual scaling improves agreement between computed and observed vibrational frequencies better than a single uniform scaling. Wong ${ }^{11}$ has looked at the performance of different density functionals and recommended scaling factors for each. As noted by Irikura et al., ${ }^{6,8,9}$ even though the uncertainty in most scaling factors is larger than typically acknowledged, empirical scaling of frequencies almost universally marks an improvement over plain harmonic ones. Nevertheless, the effectiveness of emipirical scaling is still being debated in the literature. ${ }^{22-25}$

While the works discussed above introduce uniform or separate scaling factors for different regions of the vibrational spectrum to correct harmonic vibrational frequency, Borowski's effective scaling frequency factor (ESFF) ${ }^{26-28}$ method and Pulay's scaled quantum mechanical $(\mathrm{SQM})^{29}$ force field approaches scale individual vibrational frequencies or force constants depending on the nature of the local modes (ESFF) or the internal coordinates (SQM) contributing to each vibrational mode. Both ESFF and SQM have been shown to reduce the root-mean-square (RMS) deviation between scaled vibrational frequencies and experimental frequencies impressively when applied for density functional methods with various basis sets. ${ }^{26-28,30,31}$ Even though SQM has been successfully applied to hydrogen-bonded acid dimers, ${ }^{30-33}$ its use has been limited to date.

All the empirical scaling schemes described so far are derived from training sets of small covalently bound molecules for which experimental frequencies are readily available. None of these training sets include the experimental vibrational frequencies of even water dimers: the prototypical hydrogen-bonded system with a resolved vibrational spectrum. Thus, their applicability to hydrogen-bonded systems, like water clusters, is highly questionable even though they have been routinely employed in the literature. ${ }^{34-38}$

Each water cluster $\left(\mathrm{H}_{2} \mathrm{O}\right)_{n}$ has $3 n$ high-frequency intramolecular vibrational modes corresponding to the symmetric stretch, asymmetric stretch and bending of each monomer. As water clusters form from individual monomers, the $6 n-6$ translational and rotational degrees of freedom of the monomers turn into low-frequency intermolecular vibrational modes. Experimental intramolecular vibrational frequencies are available for $\left(\mathrm{H}_{2} \mathrm{O}\right)_{n}$, however the intermolecular modes remain murky due to the coupling of lowfrequency vibrational modes with each other and rotational degrees of freedom. Moreover, the experimental spectra are taken with the water clusters in different matrices that shift and broaden the spectral lines of the clusters in ways that are difficult to interpret. Perhaps the one exception is the water dimer whose 12 experimental vibrational frequencies have been resolved with the help of theoretical calculations. ${ }^{37,39-41}$ Therefore, in the absence of reliable experimental intermolecular frequencies, it is imperative that one relies on theoretical anharmonic calculations to correct harmonic vibrational frequencies.

Many approaches have been developed to incorporate anharmonic effects in vibrational wave function calculations. One popular approach is vibrational second-order perturbation theory (VPT2) where anharmonic corrections are calculated from higher (third and fourth) order derivatives of the potential energy surface along the normal mode coordinates. The cubic and semidiagonal quartic force constants are calculated by finite differentiation of the Hessian along the normal mode coordinates. ${ }^{42,43}$ It has the advantage of being affordable (albeit substantially more expensive than a harmonic calculation), and it is often the only practical approach for most systems of interest. If one has analytical second derivatives of the energy, then the necessary third and fourth derivatives can be computed easily using finite differentiation. For a system with $N_{m}$ normal modes, the cost is $2 N_{m}+1$ times that of a single harmonic vibrational calculation. Because each of the Hessian calculations on the $2 N_{m}+1$ displaced geometries can be run separately, VPT2 calculations are amenable to parallelization. The main drawback of VPT2 is that it is subject to the problem of near degeneracies (resonances) just like all other perturbation theory.

In a manner analogous to electronic wave function methods, grid-based methods start with the vibrational self-consistent field (VSCF) approach, where each normal mode couples with all other modes in an average way. ${ }^{44-46}$ Higher order correlation between the modes is included via second-order perturbation theory (VMP2), configuration interaction (VCI), or coupledcluster (VCC) theory. These methods give good anharmonic frequencies for fundamental modes and overtones resulting from them, and they can account for coupling of different modes. They can however be expensive as they scale nonlinearly with the number of normal modes and the number of modes being correlated. ${ }^{47}$ The cost of a VPT2 calculation scales linearly with the number of normal modes, and it is typically at least an order of magnitude cheaper than VSCF and its correlation corrected analogs. Another downside of VSCF is that it often gives unreasonable anharmonic frequencies for large amplitude, lowfrequency modes, like the intermolecular modes of hydrogenbonded systems. ${ }^{48-50}$ Aside from these two popular schemes, harmonically coupled anharmonic oscillator (HCAO), ${ }^{51}$ ab initio molecular dynamics, ${ }^{52} \mathrm{P} \_$VMWCI2, ${ }^{53}$ and $\mathrm{HBB}^{54}$ have been used to account for anharmonicity in hydrogen-bonded systems.

Most of the literature on the vibrational frequencies of water clusters attempts to look at the red shift in the bonded $\mathrm{O}-\mathrm{H}$ stretching modes of gas-phase water clusters relative to the gas-phase monomer. ${ }^{36,55,56}$ Aside from the water dimer, ${ }^{57}$ there has been little work done on the effect of anharmonicity on vibrational modes of water clusters. Diri at al. ${ }^{58}$ have evaluated the effect of anharmonic correction of the binding energy and ZPVE of $\left(\mathrm{H}_{2} \mathrm{O}\right)_{n=2-6}$ using MP2 and B3LYP theories with VPT2. Dunn et al. ${ }^{37}$ showed that harmonic frequencies calculated using HF theory are more amenable to scaling than those computed using MP2. Njegic and Gordon ${ }^{50}$ have looked at the effect of the anharmonicity of vibrational modes on thermodynamic functions of small- and medium-size molecules, including the water dimer using VSCF. They concluded that vibrational anharmonicity and coupling of modes have a substantial effect on the ZPVE as well as thermodynamic functions. They suggest expressing the normal mode displacement vectors in internal instead of Cartesian coordinates to get reasonable VSCF anharmonic frequencies for modes that involve bending and torsional motion. For the case of the water dimer, casting the potential energy surface in internal coordinates improves the agreement between calculated and experimental low-frequency modes remarkably. Correlation corrected vibrational mean field theory (cc-VSCF) has also been applied to hydrogen-bonded systems. ${ }^{48,49}$ Kjaergaard at al. ${ }^{57}$ have examined the performance of VPT2, VSCF, cc-VSCF, and HCAO for the water dimer. Their results show that VSCF, cc-VSCF, and $\mathrm{HCAO}$ perform well for $\mathrm{O}-\mathrm{H}$ stretches but are somewhat erratic for intermolecular modes. VPT2 works well for all vibrational modes, even though it does not perform as well as $\mathrm{HCAO}$ for $\mathrm{O}-\mathrm{H}$ stretching frequencies. Begue et al..$^{53}$ have benchmarked different anharmonic approaches and found VPT2 to be consistently applicable to water clusters, while other approaches had many pitfalls. Similarly, Torrent-Sucarrat et al. ${ }^{59}$ have studied the role of vibrational anharmonicity in hydrogen-bonded complexes formed between the hydroperoxyl radical and formic, acetic, nitric, and sulfuric acids. They conclude that VPT2 anharmonic frequencies computed over 
B3LYP/6-31+G(d,p) and B3LYP/6-311+G(2d,2p) PES agree well with experiment. Watanabe et al. ${ }^{60}$ used a scaled hypersphere search (SHS) by polynomial fitting of the intramolecular potential energy function of water clusters to get fundamental frequencies that were very close to experimental values. A recent application of these different anharmonic treatments to $(\mathrm{HF})_{n=2-4}$ showed that VPT2, VSCF, and cc-VSCF perform comparably for high-frequency stretching modes, but the latter two were unreliable for lowfrequency intermolecular modes. ${ }^{47}$ Dykstra has looked at anharmonic effects on zero point energies of weakly bound molecular clusters including the water dimer and trimer and concluded that the effect is large when there are multiple minima on the intermolecular potential energy surface. ${ }^{61}$

Low-frequency modes are generally much more anharmonic than high-frequency ones. They also couple with each other as well as rotational degrees of freedom, thus making theoretical treatments and experimental spectral resolutions daunting. Since lowfrequency modes contribute the most to the thermal correction to the enthalpy and entropy of a system, even small deviations in these modes lead to large errors in free energies. As a result, researchers have been somewhat reluctant to use calculated harmonic frequencies to estimate free energies of weakly bound clusters, particularly at high temperatures where the errors would be most pronounced. ${ }^{62}$

On the basis of existing literature, VPT2 is the most appropriate and affordable approach for evaluating the role of anharmonicity in large water clusters. Even though recent works by Barone et al. ${ }^{63,64}$ advocate the use of density functional methods with modest basis sets to calculate VPT2 anharmonic frequencies, we chose to use an MP2 wave function because it has been shown to be the most affordable and accurate method for studying water clusters. The aug-cc-pVDZ basis set has the necessary diffuse and polarization functions to describe hydrogen bonding well, and it is often used to determine optimal geometries and harmonic vibrational frequencies. MP2 calculations with correlation consistent basis sets, when extrapolated to the CBS limit, capture all the important features of small water clusters. ${ }^{65-80} \mathrm{MP} 2 /$ aug-cc-pVDZ harmonic vibrational frequencies have been used for benchmark quality works on water clusters as large as $\left(\mathrm{H}_{2} \mathrm{O}\right)_{17}$ due to their affordability. ${ }^{80}$ Therefore, appropriate scaling factors for the MP2/aug-cc-pVDZ level of theory are especially important.

In this paper, we demonstrate the need to use separate scaling factors for intermolecular vibrational modes of hydrogen-bonded clusters. For the commonly used MP2/aug-cc-pVDZ level of theory, we provide a set of frequency scaling factors for harmonic frequencies as well as the ZPVE and vibrational corrections to the enthalpy and entropy. Using 723 VPT2 anharmonic frequencies calculated for $\left(\mathrm{H}_{2} \mathrm{O}\right)_{n=2-6,8,9}$, scaling factors for the harmonic vibrational frequencies have been determined. The intermolecular modes $\left(\omega<1100 \mathrm{~cm}^{-1}\right)$ are found to be substantially more anharmonic than intramolecular bending $\left(1100 \mathrm{~cm}^{-1}<\right.$ $\left.\omega<1800 \mathrm{~cm}^{-1}\right)$ and stretching modes $\left(\omega>1800 \mathrm{~cm}^{-1}\right)$, suggesting that the use of different frequency scaling factors for each region in correcting the harmonic vibrational modes is appropriate. Similarly, by comparing the harmonic and anharmonic ZPVE, vibrational contribution to the enthalpy $\left(\Delta H_{\mathrm{vib}}\right)$ and entropy $\left(S_{\text {vib }}\right)$, scaling factors are calculated. These different scaling approaches are applied to the water dimer system in order to evaluate the importance of anharmonicity and the validity of the rigid rotor-harmonic oscillator ( $\mathrm{RRHO}$ ) model. The transferability of the recommended scaling factors to other levels of theory and hydrogen-bonded systems is also discussed.

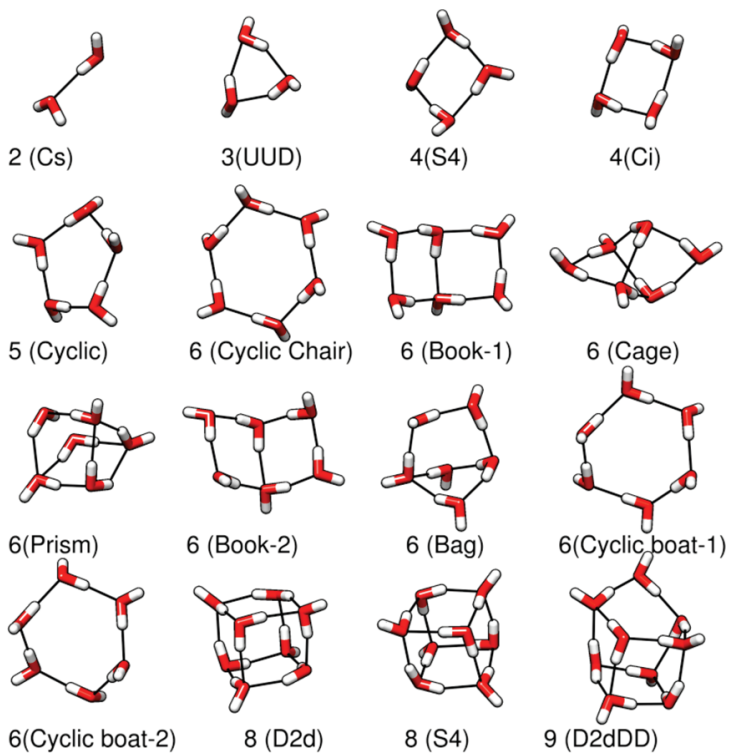

Figure 1. The 16 water clusters included in this study.

\section{METHODOLOGY}

The clusters included in this study are the water dimer $\left(2-C_{s}\right)$, trimer (3-UUD), tetramers $\left(4-S_{4}, 4-C_{i}\right)$, pentamer (5-Cyclic), hexamers (6-Cyclic-chair, 6-Book-1, 6-Cage, 6-Prism, 6-Book-2, 6-Bag, 6-Cyclic-boat-1, 6-Cyclic-boat-2), octamers $\left(8-D_{2 d}, 8-S_{4}\right)$, and one nonamer $\left(9-D_{2 d} \mathrm{DD}\right)$. The optimized structures are shown in Figure 1. These 16 clusters have a total of 723 harmonic vibrational modes. The geometry optimizations and harmonic vibrational frequencies were computed using MP2/aug-ccpVDZ with analytical gradients and Hessians. To avoid numerical problems in subsequent anharmonic frequency calculations, tight convergence criteria was enforced for both the geometry optimization and the Hessian calculations. We denote VPT2 anharmonic calculations on MP2/aug-cc-pVDZ potential energy function as VPT2/MP2/aVDZ for the sake of brevity. For these anharmonic calculations, the necessary third and fourth derivatives were determined by finite differentiation of analytic Hessians with respect to nuclear displacements along each normal mode. The default $0.0250 \AA^{43}$ step size is appropriate for rigid and semirigid systems, but it gives erratic anharmonic frequencies for the larger water clusters even after our geometries and energies were converged very tightly. A step size that gave the most reasonable anharmonic frequencies for $\left(\mathrm{H}_{2} \mathrm{O}\right)_{n=2-6,8,9}$ is $0.0050 \AA$.

One unintended consequence of using small step sizes is that it reduced the number of resonances encountered in VPT2 calculation. One of the shortcomings of VPT2 is that it suffers in handling Fermi (that affect modes coupled by cubic force constants) and Darling-Dennison resonances (which affect modes coupled by quartic force constants and Coriolis coupling constants). ${ }^{81}$ We used the default cutoffs for Fermi and Darling-Dennison resonances. Gaussian 09 A. $02^{82}$ removes resonances in an automated way as prescribed by Martin et al. ${ }^{83,84}$ All computations are performed using the Gaussian 09 A.02 ${ }^{82}$ software package on a 128-core SGI Altix $3700 \mathrm{Bx} 2$. There are differences in anharmonic frequencies calculated using Gaussian 03 B.02, ${ }^{85}$ Gaussian 09 A.02, and CFOUR ${ }^{86}$ In the interest of consistency, all our calculations are performed using Gaussian 09 A.02. ${ }^{82}$

Of the 16 clusters we studied, 4 hexamers had one or more anharmonic frequencies that are abnormally small compared to 


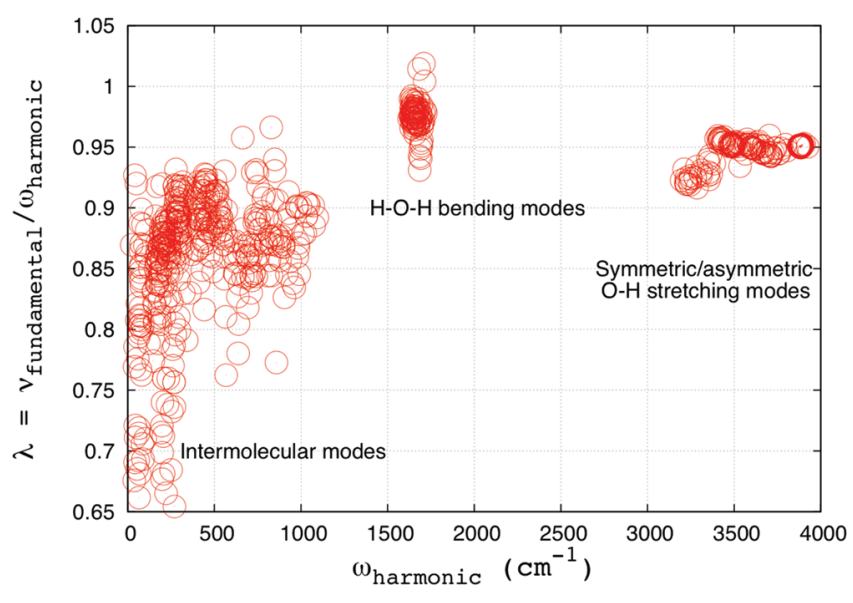

Figure 2. The differing anharmonicity in the three classes of VPT2/ MP2/aug-cc-pVDZ vibrational modes of water clusters $\left(\mathrm{H}_{2} \mathrm{O}\right)_{2-6,8,9}$.

the harmonic analog. These outliers have individual scaling factors $\left(\lambda_{i}=v_{i} / \omega_{i}\right)$ that lie outside of three standard deviations $(\sigma)$ from the average $\lambda$ of each class. Since these anomalous frequencies introduce large uncertainty, they have been removed from the scaling scheme. Scaling factors are calculated in a manner that is partly different from previous works. ${ }^{3-5,7}$ First, while others have used the inverse of frequencies to calculate scaling factors for low-frequency modes, we find such an approach to massively skew the scaling factors toward those of the lowest (and most error prone) frequency modes. Scaling the inverse of the frequency may be appropriate for covalently bonded systems where the anharmonicity rarely exceeds $10 \%$ even for low-frequency modes, but it gives unreasonably lowscaling factors and large errors when applied to the highly anharmonic intermolecular modes of our water clusters. Since we preemptively separate low-frequency intermolecular modes into their own class, deriving the frequency scaling factors using the normal frequencies works reasonably well. Second, the ZPVE scaling factors in our case are determined by scaling the harmonic ZPVE against an estimate of the true ZPVE in a manner suggested by Barone et al. ${ }^{42}$ Third, to get scaling factors for $\Delta H_{\text {vib }}$ and $S_{\text {vib, }}$ previous works used least-squares fitting of frequencies to minimize the residual between the experimental

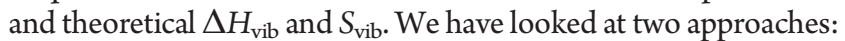
(a) scaling the harmonic $\Delta H_{\text {vib }}$ and $S_{\text {vib }}$ against their anharmonic analogs directly and (b) determining frequency scaling factors that minimize the residual of $\Delta H_{\text {vib }}$ and $S_{\text {vib. }}$. The merits and downsides of both approaches are discussed.

\section{RESULTS AND DISCUSSION}

3.1. Classification of Vibrational Frequencies. Each water cluster $\left(\mathrm{H}_{2} \mathrm{O}\right)_{n}$ has $2 n$ high-frequency intramolecular stretching modes, $n$ intramolecular bending modes, and $6 n-6$ lowfrequency intermolecular modes. In this study, there are 723 vibrational modes of which 182 correspond to monomer stretching, 91 to monomer bending, and 450 to low-frequency intermolecular motion. Removing a few anomalous anharmonic frequencies leaves us with 703 vibrational frequencies of which $435,271,88$, and 178 are intermolecular, intramolecular, bending, and stretching modes, respectively. As illustrated in Figure 2, the distribution of the ratio of anharmonic to harmonic frequencies $\left(\lambda=v^{\mathrm{f}} / \omega^{\mathrm{h}}\right)$ for the water clusters in this study shows three distinct groupings. The stretching modes which lie above $3000 \mathrm{~cm}^{-1}$ are not particularly anharmonic; as can be seen from the $\lambda \sim 0.95$, one would need to match the harmonic frequencies to the fundamentals. The bending modes are even less anharmonic, needing a scaling factor of $\lambda \sim 0.97$ to match fundamentals. In contrast, the low-frequency intermolecular modes have a larger and more spread anharmonicity. Figure 2 demonstrates the need to use separate multiplicative factors to scale the different classes of frequencies. Johnson et al. ${ }^{9}$ outline the three conditions that need to be met to form a class of frequencies as "(1) the bias $(\lambda)$ for the target frequency is believed to be of similar value to those in the class; (2) the (estimated) biases in the class have an approximately normal and acceptably narrow distribution; (3) the number of vibrational frequencies in the class is reasonably large." Our grouping of the water clusters frequencies into two or three classes satisfies the conditions listed above. The frequencies can be classified into intermolecular $\left(\omega^{\mathrm{h}}<1100 \mathrm{~cm}^{-1}\right)$ and intramolecular $\left(\omega^{\mathrm{h}}>\right.$ $1100 \mathrm{~cm}^{-1}$ ) modes, and that scheme will be designated as twosplit scaling. Alternatively, the frequencies can be grouped into intermolecular $\left(\omega^{\mathrm{h}}<1100 \mathrm{~cm}^{-1}\right)$, bending $\left(1100 \mathrm{~cm}^{-1}<\omega^{\mathrm{h}}<\right.$ $\left.1800 \mathrm{~cm}^{-1}\right)$, and stretching $\left(\omega^{\mathrm{h}}>1800 \mathrm{~cm}^{-1}\right)$ modes, in a scheme we will call three-split scaling. A comparison of uniform scaling with the two- and three-split scaling schemes is performed below.

3.2. Scaling Vibrational Frequencies. Given $N \mathrm{MP} 2 / \mathrm{aVDZ}$ harmonic frequencies $\left(\omega^{\mathrm{h}}\right)$ and VPT2/MP2/aVDZ anharmonic fundamental frequencies $\left(v^{\mathrm{f}}\right)$, an optimal scaling factor, $\lambda$ can be found by using the least-squares procedure minimizing the residual $(\Delta)$ which is defined as

$$
\Delta=\sum_{i=1}^{N}\left(\lambda \omega_{i}^{\mathrm{h}}-v_{i}^{\mathrm{f}}\right)^{2}
$$

The $\lambda$ that minimizes the residual is

$$
\lambda=\frac{\sum_{i=1}^{N} \omega_{i}^{\mathrm{h}} v_{i}^{\mathrm{f}}}{\sum_{i=1}^{N}\left(\omega_{i}^{\mathrm{h}}\right)^{2}}
$$

How well this scaling factor improves the harmonic frequencies is assessed by evaluating the root-mean-square error (RMSE) of the scaled frequency relative to the anharmonic fundamental:

$$
\begin{aligned}
\text { RMSE } & =\sqrt{\frac{1}{N} \sum_{i=1}^{N}\left(\lambda \omega_{i}^{\mathrm{h}}-v_{i}^{\mathrm{f}}\right)^{2}} \\
\text { RMSE } & =\sqrt{\frac{\Delta}{N}}
\end{aligned}
$$

The uncertainty associated with the scaling factor $\lambda$ is a critical measure of the confidence and applicability of the scaling scheme, as shown repeatedly by Irikura et al. ${ }^{6,8,9}$ The uncertainty $\sigma(\lambda)$ is defined as

$$
\sigma(\lambda)=\sqrt{\frac{\Delta}{\sum_{i=1}^{N}\left(\omega_{i}^{\mathrm{h}}\right)^{2}}}
$$

As noted already, there are many ways to calculate frequency scaling factors. Table 1 shows the scaling factors for uniform, two- and three-split scaling schemes for each separate water 
Table 1. Individual Scaling Factors for MP2/aug-cc-pVDZ Harmonic Frequencies Relative to VPT2/MP2/aug-cc-pVDZ Anharmonic Frequencies of $\left(\mathrm{H}_{2} \mathrm{O}\right)_{n=2-6,8,9}$

\begin{tabular}{|c|c|c|c|c|c|c|}
\hline & \multirow{2}{*}{$\begin{array}{l}\text { uniform } \\
\lambda \text { (all) }\end{array}$} & \multicolumn{2}{|c|}{ two-split scaling } & \multicolumn{3}{|c|}{ three-split scaling } \\
\hline & & $\lambda(\omega<1100)$ & $\lambda(\omega>1100)$ & $\lambda(\omega<1100)$ & $\lambda(1100<\omega<1800)$ & $\lambda(\omega>1800)$ \\
\hline $2-C_{s}$ & 0.954 & 0.831 & 0.955 & 0.831 & 0.972 & 0.953 \\
\hline 3-UUD & 0.951 & 0.811 & 0.955 & 0.811 & 0.970 & 0.953 \\
\hline 4- $C_{i}$ & 0.952 & 0.875 & 0.955 & 0.875 & 0.976 & 0.952 \\
\hline $4-S_{4}$ & 0.952 & 0.878 & 0.954 & 0.878 & 0.980 & 0.952 \\
\hline 5-Cyclic & 0.950 & 0.880 & 0.952 & 0.880 & 0.972 & 0.950 \\
\hline 6-Cyclic-chair ${ }^{a}$ & 0.950 & 0.876 & 0.953 & 0.876 & 0.971 & 0.951 \\
\hline 6-Book-1 & 0.950 & 0.880 & 0.953 & 0.880 & 0.979 & 0.950 \\
\hline 6-Cage & 0.948 & 0.864 & 0.951 & 0.864 & 0.974 & 0.949 \\
\hline 6-Prism & 0.947 & 0.872 & 0.950 & 0.872 & 0.983 & 0.946 \\
\hline 6-Book-2 ${ }^{a}$ & 0.947 & 0.878 & 0.949 & 0.878 & 0.969 & 0.947 \\
\hline 6-Bag & 0.947 & 0.876 & 0.950 & 0.876 & 0.975 & 0.947 \\
\hline 6-Cyclic-boat- $1^{a}$ & 0.949 & 0.864 & 0.952 & 0.864 & 0.967 & 0.951 \\
\hline 6-Cyclic-boat- $2^{a}$ & 0.949 & 0.863 & 0.952 & 0.863 & 0.964 & 0.950 \\
\hline $8-S_{4}$ & 0.944 & 0.888 & 0.946 & 0.888 & 0.982 & 0.943 \\
\hline $8-D_{2 d}$ & 0.944 & 0.885 & 0.947 & 0.885 & 0.983 & 0.943 \\
\hline 9- $\mathrm{D}_{2 d} \mathrm{DD}^{a}$ & 0.944 & 0.884 & 0.946 & 0.884 & 0.978 & 0.943 \\
\hline overall & 0.948 & 0.876 & 0.950 & 0.876 & 0.975 & 0.949 \\
\hline
\end{tabular}

Table 2. Collective Scaling Factors for MP2/aug-cc-pVDZ Harmonic Frequencies Relative to VPT2/MP2/aug-cc-pVDZ Anharmonic Frequencies of $\left(\mathrm{H}_{2} \mathrm{O}\right)_{n=2-6,8,9}{ }^{a}$

\begin{tabular}{|c|c|c|c|c|}
\hline & nature of mode & scaling factor $(\lambda)$ & uncertainty $[\sigma(\lambda)]$ & $\operatorname{RMSE}\left(\mathrm{cm}^{-1}\right)$ \\
\hline \multicolumn{5}{|c|}{ Uniform Scaling } \\
\hline$\lambda($ all $)$ & all & 0.948 & 0.019 & 39 \\
\hline \multicolumn{5}{|c|}{ Two-Split Scaling } \\
\hline$\lambda(\omega<1100)$ & intermolecular & 0.876 & 0.036 & 17 \\
\hline$\lambda(\omega>1100)$ & intramolecular & 0.950 & 0.012 & 36 \\
\hline \multicolumn{5}{|c|}{ Three-Split Scaling } \\
\hline$\lambda(\omega<1100)$ & intermolecular & 0.876 & 0.036 & 17 \\
\hline$\lambda(1100<\omega<1800)$ & intramol. bending & 0.975 & 0.010 & 17 \\
\hline$\lambda(\omega>1800)$ & intramol. stretching & 0.949 & 0.007 & 25 \\
\hline
\end{tabular}

${ }^{a}$ Using 703, 435, 271, 88, and 178 frequencies for all, intermolecular, intramolecular, bending, and stretching modes, respectively, after removing outlying frequencies from an initial set of $723,450,273,91$, and 182 modes.

cluster and all 15 clusters combined. The individual scaling factors do not vary substantially among the clusters, with the dimer and the trimer being slight outliers. The overall scaling factors are given in Table 2. Applying a uniform scaling factor of $0.948 \pm 0.019$ leads to an RMSE of $39 \mathrm{~cm}^{-1}$. It is clear that the high-frequency stretching modes are dominating this scaling factor, as evidenced by the scaling factor of $0.949 \pm 0.007$ we get just for the stretching modes alone. In the two-split scaling scheme, we get a scaling factor of $0.876 \pm 0.036$ for the 435 intermolecular modes and $0.950 \pm 0.012$ for the 271 intramolecular modes. The larger uncertainty in the intermolecular scaling factor is due to the diverse range of anharmonicity in the low-frequency modes, as can be seen in the frequency range $\omega^{\mathrm{h}}<1100 \mathrm{~cm}^{-1}$ in Figure 2 . The RMSE of the two-split scaling scheme is lower than that of the uniform scaling. In the three-split scaling, we derive values of 0.876 \pm 0.036 for the 435 intermolecular modes, $0.975 \pm 0.010$ for the 88 intramolecular bending modes, and $0.949 \pm 0.007$ for the 178 intramolecular stretching modes. The RMSE for this scheme is lower than that of the uniform and two-split scaling approaches. The distribution of the error in each scheme, defined as the difference between the scaled harmonic and anharmonic frequency, is shown in Figure 3. Figure 3a shows that the harmonic frequencies exceed anharmonic ones by as much as $260 \mathrm{~cm}^{-1}$, and the error distribution is large. Applying the scaling schemes substantially improves the agreement with the anharmonic frequencies, as illustrated in Figure $3 \mathrm{~b}$. The three-split scaling has the lowest average error and narrowest error distribution.

Our scaling factors for the intramolecular modes $(0.950 \pm 0.012)$ in the two-split scaling scheme are comparable to those in the literature which are constructed by least-squares fitting of theoretical 

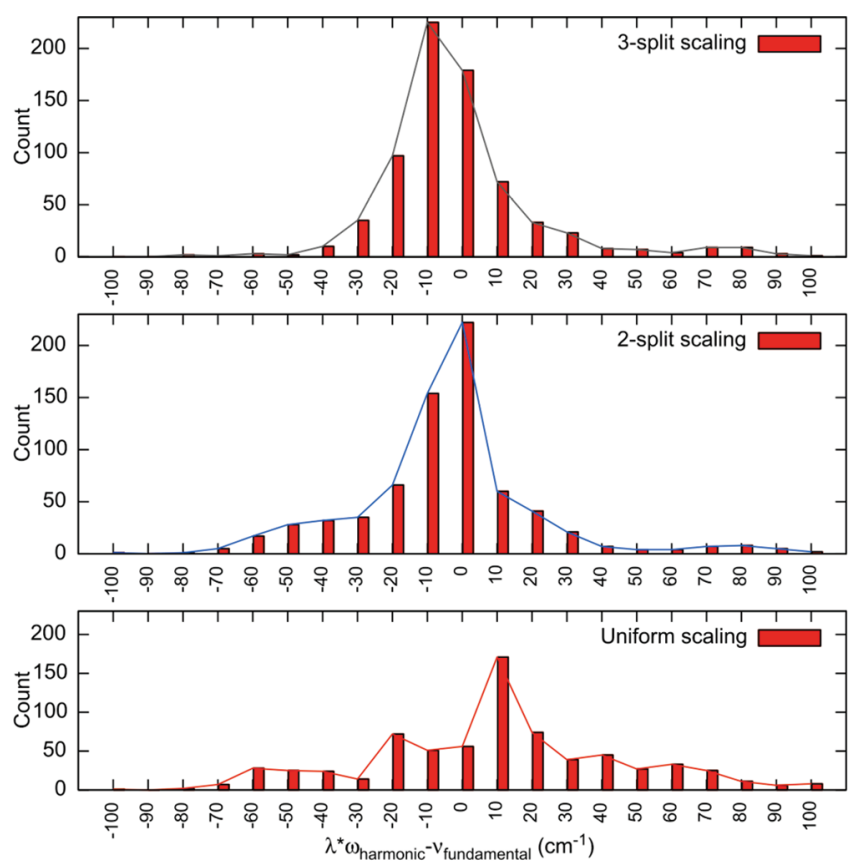

(b)

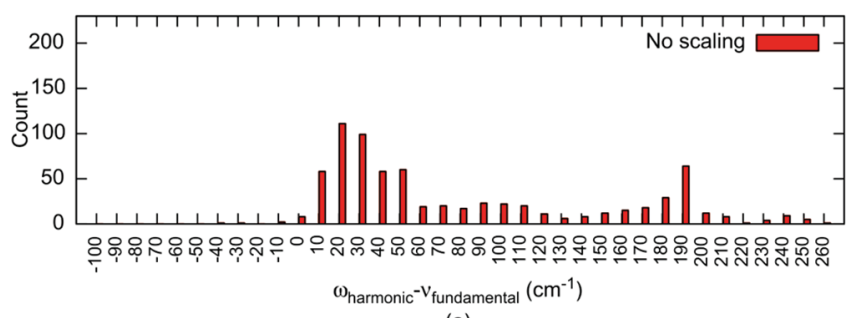

(a)

Figure 3. Error distribution of unscaled (a) and scaled (b) MP2/aVDZ harmonic vibrational frequencies of $\left(\mathrm{H}_{2} \mathrm{O}\right)_{2-6,8,9}$ relative to VPT2/ $\mathrm{MP} 2 / \mathrm{aVDZ}$ anharmonic frequencies. Please note that different abscissa scales are used in (a) and (b).

harmonic vibrational frequencies to experimental fundamental frequencies of covalently bonded molecules. For the MP2/aVDZ level of theory, Merrick et al. report scaling factors of 0.9615 and 0.9614 for fundamental frequencies using the $\mathrm{F} 1$ and $F 1^{\prime \prime}$ set of frequencies. ${ }^{7}$ Sinha et al. similarly recommend a scaling factor of 0.9604 based on a database of 41 common organic molecules. ${ }^{5}$ There are two reasons for the slight differences between our scaling factors and those from the literature. First, we are comparing harmonic frequencies against calculated anharmonic frequencies, while the literature references compare against experimental fundamental frequencies. Second, we are using a database of hydrogenbonded water clusters, while those in the literature rely on a set of small covalently bonded molecules.

The most stark difference between our scaling scheme and others is the scaling factor for the low-frequency intermolecular modes. Merrick et al. use inverse frequency scaling and obtain scaling factors of 1.0418 and 1.0338 for the $\mathrm{F} 1$ and $F 1^{\prime \prime}$ set of frequencies for the MP2/aVDZ level of theory. ${ }^{7}$ Using the same approach on a database of 41 organic molecules, Sinha at al. get scaling factors for MP2/aVDZ low frequencies $\left(\omega^{\mathrm{h}}<1000 \mathrm{~cm}^{-1}\right)$ of $1.0999 .{ }^{5}$ We did not use inverse frequency scaling because it is unduly biased toward the lowest frequency modes which are very anharmonic and error prone. For the database of covalently bonded molecules that Merrick and Sinha used, the level of anharmonicity in the lowest frequency modes is not as severe as ours. So, it would make sense to use an inverse frequency scaling factor to overcome the dominance of the high-frequency modes in the direct scaling scheme. We circumvent that dominance by grouping our frequencies into three classes and determining separate scaling factors for each class.

3.3. Scaling of ZPVE. Provided how important quantum mechanical ZPVE corrections are to most chemical systems, a proper scaling factor for ZPVEs is crucial. As demonstrated by Grev et al., ${ }^{2}$ ZPVE scaling factors are different from plain frequency scaling factors due to the presence of anharmonicity. The harmonic and fundamental ZPVE for a molecule with $N_{m}$ vibrational modes are given by

$$
\begin{aligned}
& Z P V E^{\mathrm{h}}=\frac{1}{2} \sum_{i=1}^{N_{m}} \omega_{i}^{\mathrm{h}} \\
& Z P V E^{\mathrm{f}}=\frac{1}{2} \sum_{i=1}^{N_{m}} v_{i}^{\mathrm{f}}
\end{aligned}
$$

Since our calculated anharmonic fundamental corresponds to the $v(0 \rightarrow 1)$ transition frequency and not the energy of $v(0)$, we cannot get the anharmonic ZPVE by simply plugging the fundamental frequency into the ZPVE expression in eq 6. The true ZPVE lies somewhere in between $\mathrm{ZPVE}^{\mathrm{h}}$ and $\mathrm{ZPVE}^{\mathrm{f}}$ and various approximations to it have been given in the literature. ${ }^{2,87}$ A commonly used estimate of the true ZPVE is

$$
Z P V E=\chi_{0}+\frac{1}{2}\left(Z P V E^{\mathrm{h}}+Z P V E^{\mathrm{f}}\right)-\frac{1}{4} \sum_{i=1}^{N_{m}} \chi_{i i}
$$

where $\chi_{0}$ is a small anharmonic correction and $\chi_{i i}$ are the diagonal elements of the anharmonicity matrix. With an estimate of the true ZPVE in hand, we can derive a scaling factor that maps the harmonic ZPVE to it. For a database of $N_{\text {mols }}$ molecules, the residual of the harmonic and true ZPVE is

$$
\Delta=\sum_{i=1}^{N_{\text {mols }}}\left(\lambda\left(Z P V E_{i}^{\mathrm{h}}\right)-Z P V E_{i}\right)^{2}
$$

The $\lambda$ that minimizes the residual is

$$
\lambda=\frac{\sum_{i=1}^{N_{\text {mols }}}\left(Z P V E_{i}^{\mathrm{h}}\right)\left(Z P V E_{i}\right)}{\sum_{i=1}^{N_{\text {mols }}}\left(Z P V E_{i}^{\mathrm{h}}\right)^{2}}
$$

The RMSE and uncertainty are then given by

$$
\begin{aligned}
& \text { RMSE }=\sqrt{\frac{\Delta}{N_{\text {mols }}}} \\
& \sigma(\lambda)=\sqrt{\frac{\Delta}{\sum_{i=1}^{N_{\text {mols }}\left(Z P V E_{i}^{\mathrm{h}}\right)^{2}}}}
\end{aligned}
$$

The anharmonic fundamental $\left(v_{i}^{\mathrm{f}}\right), \chi_{0}$, and $\chi_{i i}$ terms can be determined from the quadratic, cubic, and semidiagonal quartic force constants calculated using VPT2. Of the 16 clusters in our training set, we have removed 5 because they had one or more abnormally low anharmonic frequencies. For the remaining 11 water clusters, Table 3 shows the scaling factor for each cluster and the set overall. All the scaling factors are in a narrow range 
Table 3. Scaling Factors for the MP2/aug-cc-pVDZ Harmonic ZPVE of Water Clusters ${ }^{a, b}$

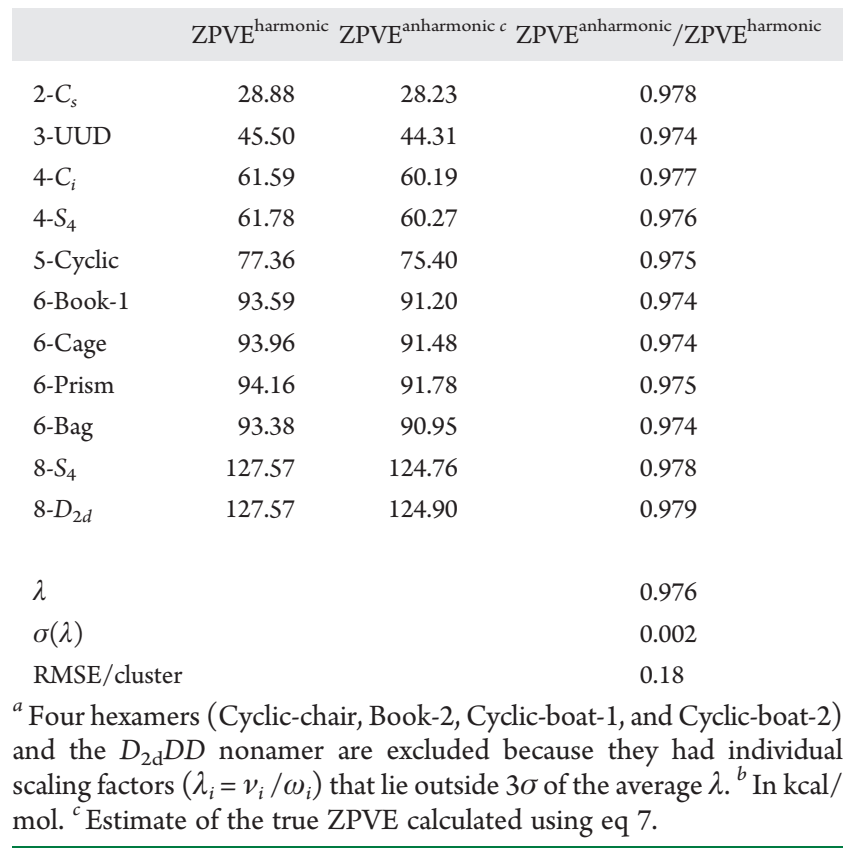

Table 4. Scaling Factors for the MP2/aug-cc-pVDZ Harmonic $\Delta H_{\mathrm{vib}}(298.15 \mathrm{~K})$ of Water ${ }^{a}$

\begin{tabular}{|c|c|c|c|}
\hline & $\begin{array}{l}\Delta H_{\mathrm{vib}}^{\mathrm{h}} \\
(298.15 \mathrm{~K})^{a}\end{array}$ & $\begin{array}{c}\Delta H_{\mathrm{vib}}^{\mathrm{f}} \\
(298.15 \mathrm{~K})^{a}\end{array}$ & $\begin{array}{l}\Delta H_{\text {vib }}^{\mathrm{f}} / \Delta H_{\text {vib }}^{\mathrm{h}} \\
(298.15 \mathrm{~K})\end{array}$ \\
\hline $2-C_{s}$ & 1.92 & 2.10 & 1.096 \\
\hline 3-UUD & 3.03 & 3.49 & 1.154 \\
\hline 4- $C_{i}$ & 4.51 & 5.01 & 1.112 \\
\hline $4-S_{4}$ & 4.40 & 4.82 & 1.096 \\
\hline 5-Cyclic & 6.27 & 6.95 & 1.109 \\
\hline 6-Book-1 & 7.74 & 8.49 & 1.096 \\
\hline 6-Cage & 7.51 & 8.39 & 1.118 \\
\hline 6-Prism & 7.46 & 8.33 & 1.117 \\
\hline 6-Bag & 7.82 & 8.70 & 1.112 \\
\hline $8-S_{4}$ & 9.75 & 10.71 & 1.098 \\
\hline $8-D_{2 d}$ & 9.75 & 10.69 & 1.097 \\
\hline \multicolumn{3}{|l|}{$\lambda_{H}$} & 1.106 \\
\hline \multicolumn{3}{|l|}{$\sigma\left(\lambda_{H}\right)$} & 0.011 \\
\hline \multicolumn{3}{|l|}{ RMSE/cluster ${ }^{b}$} & 0.07 \\
\hline \multicolumn{4}{|c|}{$\begin{array}{l}{ }^{a} \text { Four hexamers (Cyclic-chair, Book-2, Cyclic-boat-1, and Cyclic-boat-2) } \\
\text { and the } D_{2 d} \mathrm{DD} \text { nonamer are excluded because they had individual scaling } \\
\text { factors }\left(\lambda_{i}=v_{i} / \omega_{i}\right) \text { that lie outside } 3 \sigma \text { of the average } \lambda .{ }^{b} \text { In kcal } / \mathrm{mol} \text {. }\end{array}$} \\
\hline
\end{tabular}

between 0.97 and 0.98 . Our overall scaling factor of $0.976 \pm 0.002$ is close to the $0.9675^{5}$ and $0.9878^{7}$ reported in the literature from databases of small covalently bonded molecules. Considering that ZPVE is dominated by high-frequency modes, the proximity between our scaling factors and those from the literature (which have a larger number of high-frequency modes) is not surprising.

3.4. Scaling of Vibrational Corrections to the Enthalpy and Entropy. Given the vibrational energy levels of a molecule, we can calculate the vibrational partition function $Q_{v i b}(T)$ and the finite temperature vibrational correction to the enthalpy $\left[\Delta H_{\mathrm{vib}}(T)=H_{\mathrm{vib}}(T)-H_{\mathrm{vib}}(0)\right]$ and entropy $\left[S_{\mathrm{vib}}(T)\right]$. The vibrational and rotational contributions to the partition function employ the canonical rigid rotor-harmonic oscillator (RRHO) equations. ${ }^{15,88}$ In the harmonic oscillator model, the vibrational energy levels of each mode are evenly spaced, and we can get compact expressions for $Q_{v i b}(T)$ and the finite temperature corrections:

$$
\begin{aligned}
& Q_{v i b}=\prod_{i}\left(\frac{1}{1-\mathrm{e}^{-\mu_{i}}}\right) \\
& \Delta H_{v i b}(T)=R T \sum_{i}\left(\frac{\mu_{i}}{\mathrm{e}^{\mu_{i}}-1}\right) \\
& S_{v i b}(T)=R \sum_{i}\left[\frac{\mu_{i}}{\mathrm{e}^{\mu_{i}}-1}-\ln \left(1-\mathrm{e}^{-\mu_{i}}\right)\right]
\end{aligned}
$$

where $\mu_{i}=\hbar c \omega_{i} / k_{\mathrm{B}} T, R$ is the universal gas constant, $\hbar$ is Planck's constant, $c$ is the speed of light, and $\omega_{\mathrm{i}}$ is the harmonic frequency in wavenumbers. The anharmonic partition function is not amenable for such simplifications, and it has terms that include the anharmonicity constant. Anharmonic energy levels are not evenly spaced as is the case for a harmonic oscillator. One would need to sum over all the anharmonic energy levels to calculate the partition function, but the perturbation theory is prone to failures in predicting higher vibrational energy levels. To overcome these limitations, Truhlar and Isaacson ${ }^{89}$ have proposed an approximation called simple perturbation theory (SPT) which retains a form like the harmonic expression above but uses anharmonic frequencies and ZPVEs:

$$
Q_{v i b}=\frac{\exp \left(\frac{-Z P V E}{k_{\mathrm{B}} T}\right)}{\prod_{i}\left(1-\mathrm{e}^{-\mu_{i}}\right)}
$$

where $\mu_{i}=\hbar c v_{i} / k_{\mathrm{B}} T, \hbar$ is Planck's constant, $c$ is the speed of light, $v_{i}$ is the anharmonic frequency in wavenumbers, and ZPVE is the true zero point vibrational energy correction shown in eq 7. SPT has been shown to compare well with methods summing over anharmonic energy levels for linear and nonlinear molecules in the small anharmonicity limit. ${ }^{81,89-91}$ In modes like torsions, ring inversions, and internal rotations where the vibrational potential differs markedly from that of a single well harmonic oscillator, the SPT approximation will not work well. Kurtén et al. ${ }^{92}$ have recently generalized the solution to a one-dimensional system in the small anharmonicity limit to $n$-dimensions and successfully applied it to $\left(\mathrm{H}_{2} \mathrm{SO}_{4}\right)\left(\mathrm{H}_{2} \mathrm{O}\right)_{n=1-2}$ and $\left(\mathrm{HSO}_{4}{ }^{-}\right)\left(\mathrm{H}_{2} \mathrm{O}\right)_{n=1-2}$. Their more complicated anharmonic expressions contain terms including the anharmonicity constant, and they do predict vibrational enthalpies and entropies that differ substantially from those of SPT for hydrogen-bonded systems. Nevertheless, since their expressions have not been rigorously tested on a variety of systems, we will use the SPT approximation here. The SPT thermal corrections to the enthalpy and the entropy look similar to their harmonic analogs except we use the fundamental frequency $\left(\mu_{i}=\hbar c v_{i} / k_{\mathrm{B}} T\right)$ in this case:

$$
\Delta H_{v i b}(T)=R T \sum_{i}\left(\frac{\mu_{i}}{\mathrm{e}^{\mu_{i}}-1}\right)
$$




$$
S_{v i b}(T)=R \sum_{i}\left[\frac{\mu_{i}}{\mathrm{e}^{\mu_{i}}-1}-\ln \left(1-\mathrm{e}^{-\mu_{i}}\right)\right]
$$

There are two approaches to determine appropriate scaling factors for $\Delta H_{\text {vib }}(T)$ and $S_{\text {vib }}(T)$. One can calculate the harmonic and anharmonic $\Delta H_{\mathrm{vib}}(T)$ and $S_{\mathrm{vib}}(T)$ for each molecule first and seek scaling factors $\left(\lambda_{\mathrm{H}}\right.$ and $\left.\lambda_{\mathrm{S}}\right)$ that minimize the residuals:

$$
\begin{aligned}
& \Delta_{H}=\sum_{i=1}^{N_{m o l s}}\left[\lambda_{H} \Delta H_{v i b, i}\left(T, \omega^{\mathrm{h}}\right)-\Delta H_{v i b, i}\left(T, v^{\mathrm{f}}\right)\right]^{2} \\
& \Delta_{S}=\sum_{i=1}^{N_{m o l s}}\left[\lambda_{S} S_{v i b, i}\left(T, \omega^{\mathrm{h}}\right)-S_{v i b, i}\left(T, v^{\mathrm{f}}\right)\right]^{2}
\end{aligned}
$$

This method is easy to apply, and below is an analytic form for the scaling factors:

$$
\begin{gathered}
\lambda_{H}=\frac{\sum_{i=1}^{N_{m o l s}} \Delta H_{v i b, i}\left(T, \omega^{\mathrm{h}}\right) \Delta H_{v i b, i}\left(T, v^{\mathrm{f}}\right)}{\sum_{i=1}^{N_{m o l s}}\left[\Delta H_{v i b, i}\left(T, \omega^{\mathrm{h}}\right)\right]^{2}} \\
\lambda_{S}=\frac{\sum_{i=1}^{N_{m o l s}} S_{v i b, i}\left(T, \omega^{\mathrm{h}}\right) S_{v i b, i}\left(T, v^{\mathrm{f}}\right)}{\sum_{i=1}^{N_{m o l s}}\left[S_{v i b, i}\left(T, \omega^{\mathrm{h}}\right)\right]^{2}}
\end{gathered}
$$

The RMSE and uncertainty in the scaling factors are calculated simply:

$$
\begin{aligned}
& \operatorname{RMSE}_{H}=\sqrt{\frac{\Delta_{H}}{N_{\text {mols }}}} \\
& \text { RMSE }_{S}=\sqrt{\frac{\Delta_{S}}{N_{\text {mols }}}} \\
& \sigma\left(\lambda_{H}\right)=\sqrt{\frac{\sum_{i=1}^{N_{\text {mols }}\left[\Delta H_{v i b, i}\left(T, \omega^{\mathrm{h}}\right)\right]^{2}}}{\Delta_{H}}} \\
& \sigma\left(\lambda_{S}\right)=\sqrt{\frac{\sum_{i=1}^{N_{\text {mols }}\left[S_{\text {vib }, i}\left(T, \omega^{\mathrm{h}}\right)\right]^{2}}}{\Delta_{S}}}
\end{aligned}
$$

Please note that the thermal correction to the enthalpy here excludes the ZPVE, since the ZPVE has been scaled separately in the previous subsection. Here $\Delta H_{\text {vib }}(T)=H_{\text {vib }}(T)-H_{\text {vib }}(0)$. Since the ZPVE makes up a large part of the $H_{\text {vib }}(T)$ at most relevant temperatures, scaling $H_{\text {vib }}(T)$ would closely resemble scaling of the ZPVE. Thus, we are scaling the vibrational correction to the enthalpy excluding the ZPVE.

\begin{tabular}{|c|c|c|c|}
\hline \multicolumn{4}{|c|}{$S_{\text {vib }}^{\mathrm{h}}(298.15 \mathrm{~K})^{b} S_{\text {vib }}^{\mathrm{f}}(298.15 \mathrm{~K})^{b} S_{\text {vib }}^{\mathrm{f}}(298.15 \mathrm{~K}) / S_{\mathrm{vib}}^{\mathrm{h}}(298.15 \mathrm{~K})$} \\
\hline $2-C_{s}$ & 12.17 & 13.95 & 1.147 \\
\hline 3-UUD & 17.59 & 21.24 & 1.207 \\
\hline 4- $C_{i}$ & 29.16 & 33.16 & 1.137 \\
\hline $4-S_{4}$ & 27.37 & 30.63 & 1.119 \\
\hline 5-Cyclic & 43.06 & 50.06 & 1.163 \\
\hline 6-Book-1 & 52.37 & 59.84 & 1.143 \\
\hline 6-Cage & 48.19 & 56.21 & 1.166 \\
\hline 6-Prism & 47.44 & 55.44 & 1.169 \\
\hline 6-Bag & 52.94 & 62.54 & 1.181 \\
\hline $8-S_{4}$ & 61.33 & 69.49 & 1.133 \\
\hline $8-D_{2 d}$ & 61.20 & 68.87 & 1.125 \\
\hline \multicolumn{3}{|l|}{$\lambda_{\mathrm{s}}$} & 1.150 \\
\hline \multicolumn{3}{|l|}{$\sigma\left(\lambda_{\mathrm{S}}\right)$} & 0.021 \\
\hline \multicolumn{3}{|l|}{ RMSE } & 0.945 \\
\hline \multicolumn{4}{|c|}{$\begin{array}{l}{ }^{a} \text { Four hexamers (Cyclic-chair, Book-2, Cyclic-boat- } 1 \text {, and Cyclic-boat- } \\
\text { 2) and the } D_{2 d} \mathrm{DD} \text { nonamer are excluded because they had individual } \\
\text { scaling factors }\left(\lambda_{i}=v_{i} / \omega_{i}\right) \text { that lie outside } 3 \sigma \text { of the average } \lambda .{ }^{b} \text { In cal } \\
\text { mol/K. }\end{array}$} \\
\hline
\end{tabular}

Table 4 shows the scaling factors for $\Delta H_{\text {vib }}(T)$ determined using the procedure above. With the exception of the water trimer, the ratio of anharmonic to harmonic $\Delta H_{\mathrm{vib}}(298.15 \mathrm{~K})$ is between 1.09 and 1.11. A scaling factor exceeding unity makes sense here because harmonic vibrational frequencies and $\Delta H_{\text {vib }}(T)$ have an inverse relationship; as we scale down the harmonic frequencies,
Table 5. Scaling Factors for the MP2/aug-cc-pVDZ Harmonic $S_{\text {vib }}(298.15 \mathrm{~K})$ of Water Clusters ${ }^{a, b}$

the $\Delta H_{\text {vib }}(T)$ is scaled up. The overall scaling factor of $1.106 \pm$ 0.011 works well as evidenced by the RMSE of $0.07 \mathrm{kcal} / \mathrm{mol}$ for the 11 clusters in this study. Table 5 shows the scaling factor for $S_{\text {vib }}(298.15 \mathrm{~K})$ for the individual clusters and the collective group. All the scaling factors lie within the range of 1.12-1.21. Considering how sensitive the entropy is to the low-frequency modes (which are scaled down by 0.876 ), it should not come as a surprise that the entropy is scaled up by $10-20 \%$. The overall scaling factor for this scheme is $1.150 \pm 0.021$ with an RMSE of $0.95 \mathrm{cal} / \mathrm{mol} / \mathrm{K}$.

The approach just outlined for scaling $\Delta H_{\text {vib }}(T)$ and $S_{\text {vib }}(T)$ has the advantage that it gives a simple multiplicative factor that can be applied directly to harmonic $\Delta H_{\mathrm{vib}}(T)$ and $S_{\mathrm{vib}}(T)$. An alternative approach that has been advocated in the literature seeks frequency scaling factors that minimize the residuals:

$$
\begin{aligned}
& \Delta_{H}=\sum_{i=1}^{N}\left[\Delta H_{v i b}\left(T, \lambda_{H} \omega_{i}^{\mathrm{h}}\right)-\Delta H_{v i b}\left(T, v_{i}^{\mathrm{f}}\right)\right]^{2} \\
& \Delta_{S}=\sum_{i=1}^{N}\left[S_{v i b}\left(T, \lambda_{S} \omega_{i}^{\mathrm{h}}\right)-S_{v i b}\left(T, v_{i}^{\mathrm{f}}\right)\right]^{2}
\end{aligned}
$$

There is no analytic expression for the scaling factors, and they have to be determined numerically. The RMSE and uncertainty in the scaling factors are calculated in the same way as frequencies and ZPVEs. Along the same lines, it would make sense to separate the vibrational modes into three classes and scale each group independently. However, the vibrational corrections to both the enthalpy and entropy are overwhelmingly dominated by the low-frequency modes. Figure 4 shows a plot of $\Delta H_{\text {vib }}(298.15 \mathrm{~K})$ and $S_{\text {vib }}(298.15 \mathrm{~K})$ as a function of vibrational frequency. Vibrational modes of frequency exceeding $1100 \mathrm{~cm}^{-1}$ (i.e., intramolecular modes) make a very minimal contribution to $\Delta H_{\text {vib }}(298.15 \mathrm{~K})$ and $S_{\text {vib }}(298.15 \mathrm{~K})$. The contribution of the intramolecular modes to the enthalpy and entropy for small water clusters is typically around $1 \%$, which is below $0.1 \mathrm{kcal} /$ 
mol. So, it is not necessary to separate the frequencies into classes and scale them differently.

Since low-frequency modes are the dominant contributors to $\Delta H_{\text {vib }}$ and $S_{\text {vib }}$, the frequency scaling factors that minimize $\Delta H_{\text {vib }}$ and $S_{\text {vib }}$ residuals are similar to the scaling factor for lowfrequency modes. Tables 6 and 7 show the frequency scaling factors for $\Delta H_{\mathrm{vib}}$ and $S_{\mathrm{vib}}$ at temperatures ranging from $50 \mathrm{~K}$ to $373.15 \mathrm{~K}$. At low temperatures, there is only enough thermal energy to populate all the ground vibrational levels of all the modes and the excited vibrational levels of the low-frequency modes. So, we see $\Delta H_{\text {vib }}$ and $S_{\text {vib }}$ frequency scaling factors that reflect the scaling factor for low-frequency modes (0.876). As more thermal energy is available at higher temperatures, the excited vibrational levels of more modes start contributing, and the $\Delta H_{\text {vib }}$ and $S_{\text {vib }}$ frequency scaling factors increase. At temperatures below $373.15 \mathrm{~K}$, the $\Delta H_{\text {vib }}$ and $S_{\text {vib }}$ frequency scaling factors are less than 0.870 and 0.845 , respectively.

The uncertainty in the $\Delta H_{\mathrm{vib}}$ frequency scaling factors is large, particularly at low temperatures, but the RMSE is very small. For the $S_{\text {vib }}$ frequency scaling factors, both the uncertainty and the RMSE are small. Our scaling factors differ significantly from those in the literature which are intended for covalently bonded systems. Unlike our $\Delta H_{\text {vib }}(298.15 \mathrm{~K})$ frequency scaling factor of $0.866 \pm 0.040$, Sinha et al. ${ }^{5}$ and Merrick et al. ${ }^{7}$ report values of 0.9473 and 1.0359 , respectively. Our $S_{\text {vib }}(298.15 \mathrm{~K})$ frequency scaling factor of $0.841 \pm 0.016$ is different from Sinha et al.'s ${ }^{5}$ 0.9049 and Merrick et al.' ${ }^{7}$ 1.0452, respectively. Our RMSE is in general comparable to the two cited above.

The two approaches we have used to scale $\Delta H_{\mathrm{vib}}$ and $S_{\mathrm{vib}}$ (scaling $\Delta H_{\mathrm{vib}}$ and $S_{\mathrm{vib}}$ themselves or the vibrational frequencies that enter the $\Delta H_{\text {vib }}$ and $S_{\text {vib }}$ expressions) are equivalent, but scaling the frequencies is advocated in this case because that approach is more rigorous. It also happens to be the approach taken by others in the literature. $4,5,7$

3.5. Assessment of the Scaling Factors. The performance of the scaling factors reported above is evaluated in two ways. First, the vibrational scaling factors are applied to the water dimer, and the resulting vibrational frequencies are compared with experimental, harmonic, and anharmonic frequencies. While the small RMSEs reported in Table 2 and the narrow error distribution shown in

Table 6. Frequency Scaling Factors for $\Delta H_{\text {vib }}(T)$ for Water Clusters at the MP2/aug-cc-pVDZ Level of Theory ${ }^{a}$

$\begin{array}{lccc}T(\mathrm{~K}) & \text { scaling factor }\left(\lambda_{H}\right) & \text { uncertainty }\left[\sigma\left(\lambda_{H}\right)\right] & \text { RMSE }(\mathrm{kcal} / \mathrm{mol}) \\ 50 & 0.806 & 0.117 & 0.00 \\ 100 & 0.835 & 0.087 & 0.00 \\ 150 & 0.851 & 0.069 & 0.01 \\ 200 & 0.860 & 0.055 & 0.01 \\ 250 & 0.865 & 0.045 & 0.01 \\ 273.15 & 0.866 & 0.042 & 0.01 \\ 298.15 & 0.868 & 0.039 & 0.01 \\ 300 & 0.868 & 0.039 & 0.01 \\ 350 & 0.871 & 0.034 & 0.01 \\ 373.15 & 0.872 & 0.033 & 0.01\end{array}$

${ }^{a}$ Using 703 frequencies after removing outliers from an initial set of 723 frequencies.

Table 7. Frequency Scaling Factors for $S_{\text {vib }}(T)$ for Water Clusters at the MP2/aug-cc-pVDZ Level of Theory ${ }^{a}$

$\begin{array}{lccc}T(\mathrm{~K}) & \text { scaling factor }\left(\lambda_{S}\right) & \text { uncertainty }\left[\sigma\left(\lambda_{S}\right)\right] & \text { RMSE }(\mathrm{cal} / \mathrm{mol} / \mathrm{K}) \\ 50 & 0.790 & 0.040 & 0.06 \\ 100 & 0.815 & 0.028 & 0.08 \\ 150 & 0.828 & 0.023 & 0.09 \\ 200 & 0.836 & 0.020 & 0.10 \\ 250 & 0.841 & 0.018 & 0.10 \\ 273.15 & 0.843 & 0.017 & 0.11 \\ 298.15 & 0.844 & 0.016 & 0.11 \\ 300 & 0.844 & 0.016 & 0.11 \\ 350 & 0.847 & 0.015 & 0.11 \\ 373.15 & 0.848 & 0.014 & 0.11\end{array}$

${ }^{a}$ Using 703 frequencies after removing outliers from an initial set of 723 frequencies.

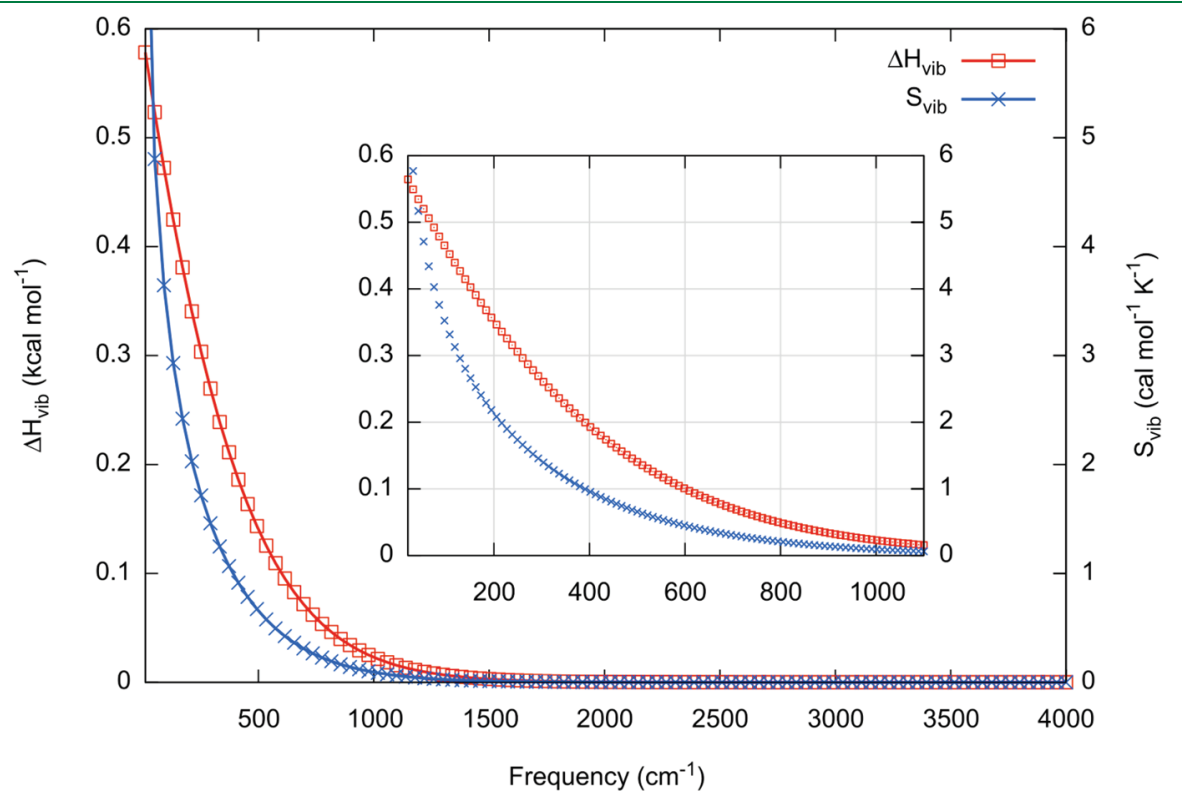

Figure 4. $\Delta H_{\mathrm{vib}}$ and $S_{\mathrm{vib}}$ as a function of frequency at $T=298.15 \mathrm{~K}$. Low-frequency modes contribute most greatly to $\Delta H_{\mathrm{vib}}\left(\right.$ left axis) and $S_{\mathrm{vib}}$ (right axis). 
Table 8. Comparison of Calculated Harmonic, ${ }^{a}$ Scaled Harmonic, ${ }^{b-d}$ and Anharmonic ${ }^{e}$ Frequencies $^{f}$ with Experimental $^{g}$ Values for the Water Dimer

\begin{tabular}{|c|c|c|c|c|c|c|}
\hline \multirow[b]{2}{*}{$\operatorname{mode}^{h}$} & \multirow[b]{2}{*}{ harmonic $^{a}$} & \multicolumn{3}{|c|}{ scaled } & \multirow[b]{2}{*}{ anharmonic $^{e}$} & \multirow[b]{2}{*}{ experiment $t^{g}$} \\
\hline & & uniform $^{b}$ & two-split $^{c}$ & three-split $^{d}$ & & \\
\hline donor torsion & 127 & 121 & 112 & 112 & 108 & 88 \\
\hline acceptor wag & 148 & 140 & 130 & 130 & 123 & 103 \\
\hline acceptor twist & 151 & 143 & 132 & 132 & 124 & 108 \\
\hline intermol. stretch & 184 & 174 & 161 & 161 & 152 & 143 \\
\hline in-plane bend & 358 & 339 & 313 & 313 & 328 & 311 \\
\hline out-of-plane bend & 639 & 605 & 560 & 560 & 514 & 523 \\
\hline$v_{2}(\mathrm{a})$ & 1624 & 1539 & 1543 & 1583 & 1581 & 1599 \\
\hline$v_{2}(\mathrm{~d})$ & 1643 & 1557 & 1561 & 1601 & 1593 & 1616 \\
\hline$v_{1}(\mathrm{~d})$ & 3704 & 3511 & 3520 & 3515 & 3554 & 3601 \\
\hline$v_{1}(\mathrm{a})$ & 3796 & 3597 & 3607 & 3602 & 3614 & 3660 \\
\hline$v_{3}(\mathrm{~d})$ & 3904 & 3700 & 3710 & 3705 & 3720 & 3735 \\
\hline$v_{3}(\mathrm{a})$ & 3925 & 3720 & 3730 & 3725 & 3730 & 3745 \\
\hline RMSE & 98 & 53 & 41 & 36 & 25 & \\
\hline
\end{tabular}

${ }^{a} \mathrm{MP} 2$ /aug-cc-pVDZ harmonic frequencies. ${ }^{b} \mathrm{MP} 2 /$ aug-cc-pVDZ harmonic frequencies scaled by $0.948 .{ }^{c} \mathrm{MP} 2 /$ aug-cc-pVDZ harmonic frequencies scaled by 0.876 and 0.950 for the inter- and intramolecular modes, respectively. ${ }^{d} \mathrm{MP} 2 /$ aug-cc-pVDZ harmonic frequencies scaled by $0.876,0.975$, and 0.949 for the intermolecular, bending, and stretching modes, respectively. ${ }^{e}$ VPT2/MP2/aug-cc-pVDZ anharmonic frequencies. ${ }^{f}$ In cm ${ }^{-1} .{ }^{g}$ See ref 37 and references therein. ${ }^{h} v_{1}$ for symmetric stretching; $v_{2}$ for bending; and $v_{3}$ for asymmetric stretching. The use of (a) signifies hydrogen-bond acceptor water, and the (d) signifies the hydrogen-bond donor water.

Figure 3 speak to the reliability of our scaling approach, a comparison with experimental frequencies answers questions about the validity of using calculated anharmonic frequencies as proxies for experimental ones. Second, the ZPVE, $\Delta H_{\text {vib }}$, and $S_{\text {vib }}$ scaling factors are applied to 11 water clusters, and their effectiveness in reproducing anharmonic numbers is assessed.

3.5.1. Vibrational Scaling Factors Applied to $\left(\mathrm{H}_{2} \mathrm{O}\right)_{2}$. The water dimer is a prototypical hydrogen-bonding system that has been the subject of extensive theoretical and experimental investigations. It remains the only water cluster for which all the experimental vibrational frequencies are available. Thus, it serves as an ideal system for assessing the performance of the harmonic, anharmonic, and scaled harmonic approaches outlined in Section 3.2.

A comparison of the theoretical harmonic and anharmonic frequencies and intensities of the water dimer against the experimental analogs has been performed by the research groups of Shields, ${ }^{37}$ Jordan, ${ }^{58}$ and Kjaergaard ${ }^{57}$ among others. We perform a similar assessment on the MP2/aVDZ frequencies and scaling schemes in Table 8 . The RMSE in the harmonic frequencies relative to experiment is large $\left(98 \mathrm{~cm}^{-1}\right)$, but scaling it by different factors brings sizable improvements. As discussed earlier, using uniform scaling factors does not correct for the varying anharmonicity in the harmonic frequencies. Using the two- and three-split scalings gives substantially better agreement with experiment, with RMSEs of 41 and $36 \mathrm{~cm}^{-1}$, respectively. The experimental frequencies are closer to the anharmonic frequencies $\left(\mathrm{RMSE}=25 \mathrm{~cm}^{-1}\right)$ than they are to the harmonic ones $\left(\mathrm{RMSE}=98 \mathrm{~cm}^{-1}\right)$. That observation further validates our decision to use calculated anharmonic frequencies as proxies for experimental frequencies. In the case of larger water clusters whose vibrational spectra are not resolved fully, calculated anharmonic frequencies serve an indispensible role.

3.5.2. $Z P V E, \Delta H_{\text {vib, }}$ and $\Delta S_{\text {vib }}$ Scaling Factors Applied to $\left(\mathrm{H}_{2} \mathrm{O}\right)_{n=2-6,8}$. Here, we want to evaluate: (a) the ability of our scaling factors to map harmonic values onto anharmonic ones and (b) the magnitude of the anharmonic correction to the ZPVE,
$\Delta H_{\text {vib, }}$ and $S_{\text {vib. }}$. The scaled harmonic ZPVE is calculated by multiplying the harmonic ZPVE by 0.976 (see Table 3), while the harmonic and anharmonic values are determined using eqs 5 and 7 , respectively. For $\Delta H_{\text {vib }}$ and $S_{\text {vib, the harmonic and }}$ anharmonic values are computed using eqs 16 and 17 . The scaled harmonic values require proper frequency factors for $T=298.15 \mathrm{~K}$. Looking at Tables 6 and 7, those factors are $\lambda_{H}=0.868$ and $\lambda_{S}=$

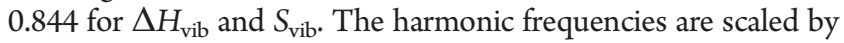
these factors before being input into eqs 16 and 17 to get the scaled harmonic $\Delta H_{\text {vib }}$ and $S_{\text {vib. }}$

Figure $5 \mathrm{a}-\mathrm{c}$ displays a comparison of ZPVE, $\Delta H_{\mathrm{vib}}(298.15 \mathrm{~K})$, and $S_{\text {vib }}(298.15 \mathrm{~K})$ for 11 water clusters. It is quite evident that application of our scaling factors works remarkably well in mapping the harmonic values to their anharmonic analogs. Starting with the ZPVE, the largest absolute difference between the scaled harmonic and anharmonic value is only $0.39 \mathrm{kcal} / \mathrm{mol}$ for the $S_{4}$ octamer. This difference is small compared to the largest absolute difference between the harmonic and anharmonic ZPVE, which is $2.82 \mathrm{kcal} / \mathrm{mol}$ for the same $S_{4}$ octamer. The absolute difference between the scaled harmonic and anharmonic ZPVE ranges from 0.05 to $0.39 \mathrm{kcal} / \mathrm{mol}$, while that between the harmonic and anharmonic values is 0.65 to $2.82 \mathrm{kcal} / \mathrm{mol}$. Considering the magnitude of the ZPVE spans $\sim 28 \mathrm{kcal} / \mathrm{mol}$ for the water dimer to $\sim 125 \mathrm{kcal} / \mathrm{mol}$ for the octamer, the scaled harmonic value is matching the anharmonic one within $0.5 \%$ or less.

For $\Delta H_{\text {vib }}(298.15 \mathrm{~K})$, we again see that our scaled harmonic value agrees remarkably well with the anharmonic value. The absolute difference between the scaled harmonic and anharmonic values ranges from 0.01 to $0.16 \mathrm{kcal} / \mathrm{mol}$, while that between the harmonic and anharmonic values is 0.18 to $0.96 \mathrm{kcal} / \mathrm{mol}$. Likewise, our scaled harmonic $S_{\text {vib }}(298.15 \mathrm{~K})$ is in great agreement with the anharmonic values. The absolute difference between the scaled harmonic and anharmonic value is in the range of $0.01-2.68 \mathrm{cal} / \mathrm{mol} / \mathrm{K}$, while the harmonic and anharmonic values differ by as much as $9.60 \mathrm{cal} / \mathrm{mol} / \mathrm{K}$. 


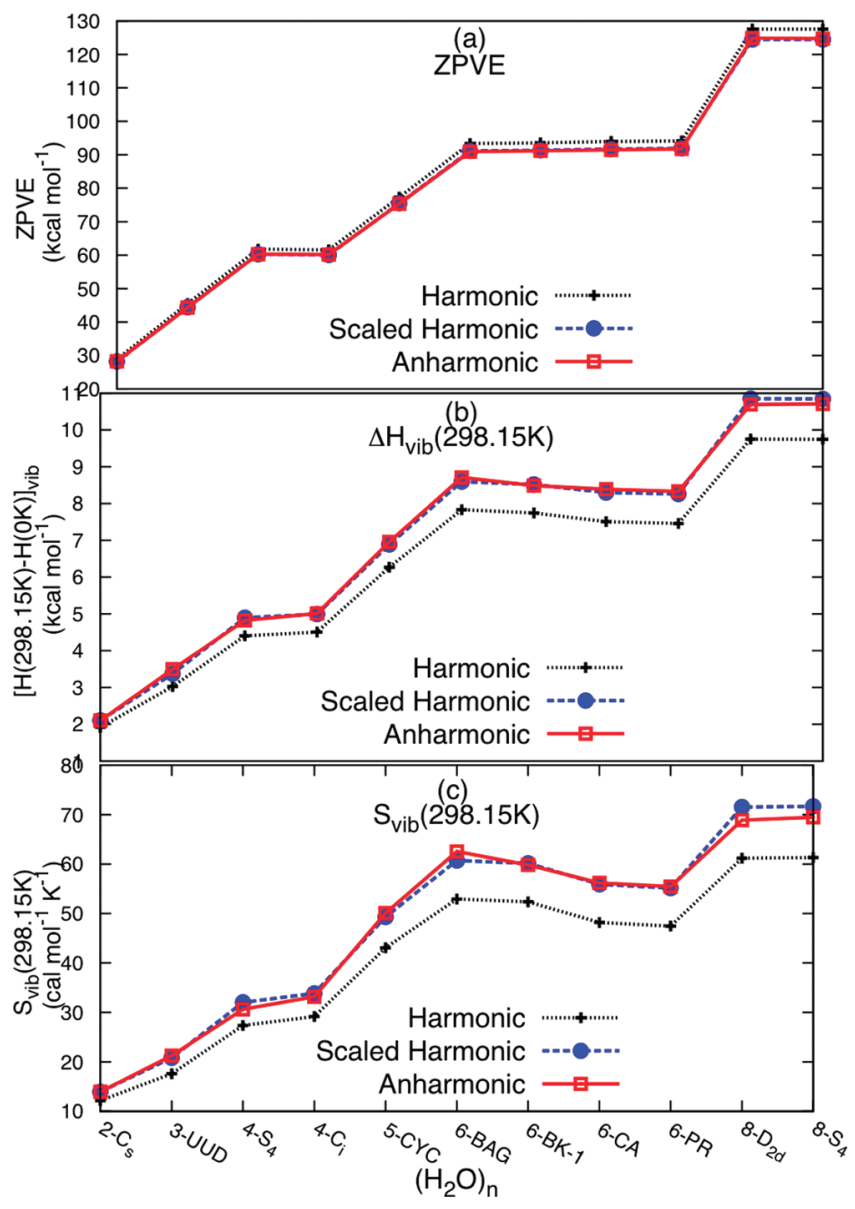

Figure 5. Comparison of harmonic, scaled harmonic and anharmonic ZPVE(a), $\Delta H_{\text {vib }}\left(\right.$ b) and $S_{\text {vib }}($ c) for 11 water clusters at $298.15 \mathrm{~K}$. The harmonic and anharmonic frequencies are calculated using [VPT2]/ MP2/aug-cc-pVDZ level of theory. We used a scaling factor of 0.976 to get the scaled harmonic ZPVE. The scaled harmonic $\Delta H_{\text {vib }}$ and $S_{\text {vib }}$ are calculated using harmonic vibrational frequencies scaled by 0.868 and 0.844 , respectively.

The apparent gap between the harmonic and anharmonic values for ZPVE and $\Delta H_{\text {vib }}$ and $S_{\text {vib }}$ in Figure 5 is indicative of the importance of anharmonicity. The anharmonic correction, which we define as the difference between the harmonic and anharmonic values as a percentage of the anharmonic value, is $1.8-2.7 \%$ for the ZPVE, $8.7-13.4 \%$ for the $\Delta H_{\text {vib }}$, and $10.7-17.2 \%$ for the $S_{\text {vib. }}$ Given such large anharmonic corrections, it is very important that one accounts for them. More importantly, when scaling factors are used to correct for anharmonicity, it is essential that the proper scaling factors are used. Applying scaling factors intended for covalently bonded systems to hydrogen-bonded clusters could lead to large errors, even though we have not attempted to quantify them here.

3.6. Transferability of the Scaling Factors. The vibrational scaling factors we have determined are strictly intended for application to the MP2/aVDZ harmonic vibrational frequencies of water clusters. Even though our training set contains vibrational frequencies for $\left(\mathrm{H}_{2} \mathrm{O}\right)_{n=2-6,8,9}$, the scaling factors should be applicable to larger water clusters. At the very least, they should perform better than conventional frequency scaling factors that are based on training sets of covalently bonded systems. The transferability of these scaling factors to other hydrogen-bonded systems requires further investigation. Our preliminary look at sulfuric acid hydrates, $\mathrm{H}_{2} \mathrm{SO}_{4}\left(\mathrm{H}_{2} \mathrm{O}\right)_{n=1-2}$ yielded reasonable VPT2 fundamental frequencies (see Tables S17 and S18 of the Supporting Information) indicating that a scaling scheme similar to that we devised for water clusters can be developed. However, it is not as trivial to partition the vibrational frequencies into physically meaningful classes (such as intramolecular stretching, intramolecular bending, and intermolecular modes) as it is for water clusters. That is because nine of $\mathrm{H}_{2} \mathrm{SO}_{4}$ vibrational modes lie below $1000 \mathrm{~cm}^{-1}$, which is in the same region as the hydrogen-bonded intermolecular modes. Thus, one would need to develop an algorithm to determine whether certain low-frequency modes are primarily intramolecular $\mathrm{H}_{2} \mathrm{SO}_{4}$ modes or intermolecular modes. While such an approach is certainly possible, it borders on the territory of other scaling methods like SQM and ESFF which are better equipped to handle such cases.

\section{CONCLUSION}

Using a training set of 16 water clusters with a combined 723 vibrational frequencies, we have determined scaling factors for vibrational frequencies, ZPVEs, $\Delta H_{\text {vib }}(T)$, and $S_{\text {vib }}(T)$ at the MP2/aug-cc-pVDZ level of theory. Our scaling factors were determined by comparing harmonic vibrational frequencies with VPT2 anharmonic fundamentals. For vibrational frequencies, it is important to separate the modes into different classes because of the varying range of anharmonicities. The disparity between our scaling factors and those derived from databases of covalently bonded systems highlights the need to use different scaling factors for hydrogen-bonded systems. The application of our scaling factors to the water dimer binding energy illustrates the importance of accounting for anharmonic effects. Our scaling factors can readily be applied to calculations on water clusters using the MP2/aug-cc-pVDZ level of theory, but their applicability to other hydrogen-bonded systems has yet to be tested. While VPT2 or the three-split scaling methods are the most reliable techniques for calculating anharmonic frequencies, the relatively good agreement using a single scaling factor supports the conclusions obtained from past studies of water cluster free energies. ${ }^{93,94}$

\section{ASSOCIATED CONTENT}

S Supporting Information. All the MP2/aVDZ optimized geometries, energies, VPT2/MP2/aVDZ harmonic and fundamental frequencies for $\left(\mathrm{H}_{2} \mathrm{O}\right)_{n=2-6,8,9}$ and $\mathrm{H}_{2} \mathrm{SO}_{4}\left(\mathrm{H}_{2} \mathrm{O}\right)_{n=1-2}$. This information is available free of charge via the Internet at http://pubs.acs.org/.

\section{AUTHOR INFORMATION}

\section{Corresponding Author}

*E-mail: george.shields@bucknell.edu.

\section{ACKNOWLEDGMENT}

Acknowledgment is made to the NSF and Bucknell University for their support of this work. This project was supported in part by NSF grant CHE-0848827 and by NSF grants CHE-0116435, CHE-0521063, and CHE-0849677 as part of the MERCURY high-performance computer consortium (http://mercuryconsortium.org). We thank Drs. Theo Kurten and Madis Noppel for helpful discussions and the reviewers for useful comments. 


\section{REFERENCES}

(1) Hehre, W. J. Ab Initio Molecular Orbital Theory; Wiley: New York, 1986.

(2) Grev, R. S.; Janssen, C. L.; Schaefer, H. F., III Concerning Zeropoint Vibrational Energy Corrections to Electronic Energies. J. Chem. Phys. 1991, 95, 5128.

(3) Pople, J.; Scott, A.; Wong, M.; Radom, L. Scaling Factors For Obtaining Fundamental Vibrational Frequencies And Zero-Point Energies From HF/6-31G* And MP2/6-31G* Harmonic Frequencies. Isr. J. Chem. 1993, 33, 345.

(4) Scott, A. P.; Radom, L. Harmonic Vibrational Frequencies: An Evaluation of Hartree-Fock, Moller-Plesset, Quadratic Configuration Interaction, Density Functional Theory, and Semiempirical Scale Factors. J. Phys. Chem. 1996, 100, 16502.

(5) Sinha, P.; Boesch, S. E.; Gu, C. M.; Wheeler, R. A.; Wilson, A. K. Harmonic Vibrational Frequencies: Scaling Factors for HF, B3LYP, and MP2Methods in Combination with Correlation Consistent Basis Sets. J. Phys. Chem. A 2004, 108, 9213.

(6) Irikura, K. K.; Johnson, R. D.; Kacker, R. N. Uncertainties in Scaling Factors for ab Initio Vibrational Frequencies. J. Phys. Chem. A 2005, 109, 8430.

(7) Merrick, J. P.; Moran, D.; Radom, L. An Evaluation of Harmonic Vibrational Frequency Scale Factors. J. Phys. Chem. A 2007, 111, 11683.

(8) Irikura, K. K.; Johnson, R. D., III; Kacker, R. N.; Kessel, R. Uncertainties in Scaling Factors for Ab Initio Vibrational Zero-point Energies. J. Chem. Phys. 2009, 130, 114102.

(9) Johnson, R. D., III; Irikura, K. K.; Kacker, R. N.; Kessel, R. Scaling Factors and Uncertainties for ab Initio Anharmonic Vibrational Frequencies. J. Chem. Theory Comput. 2010, 6, 2822.

(10) Alecu, I. M.; Zheng, J.; Zhao, Y.; Truhlar, D. G. Computational Thermochemistry: Scale Factor Databases and Scale Factors for Vibrational Frequencies Obtained from Electronic Model Chemistries. J. Chem. Theory Comput. 2010, 6, 2872.

(11) Wong, M. W. Vibrational Frequency Prediction using Density Functional Theory. Chem. Phys. Lett. 1996, 256, 391.

(12) Halls, M. D.; Velkovski, J.; Schlegel, H. B. Harmonic Frequency Scaling Factors for Hartree-Fock, S-VWN, B-LYP, B3-LYP, B3-PW91 and MP2 with the Sadlej pVTZ Electric Property Basis Set. Theor. Chem. Acc. 2001, 105, 413.

(13) Pitzer, K. S.; Gwinn, W. D. Energy Levels and Thermodynamic Functions for Molecules with Internal Rotation I. Rigid Frame with Attached Tops. J. Chem. Phys. 1942, 10, 428.

(14) Ayala, P. Y.; Schlegel, H. B. Identification and Treatment of Internal Rotation in Normal Mode Vibrational Analysis. J. Chem. Phys. 1998, 108, 2314.

(15) East, A. L. L.; Radom, L. Ab initio Statistical Thermodynamical Models for the Computation of Third-law Entropies. J. Chem. Phys. 1997, 106, 6655.

(16) Katzer, G.; Sax, A. F. Identification and Thermodynamic Treatment of Several Types of Large-amplitude Motions. J. Comput. Chem. 2005, 26, 1438.

(17) Fabian, W. M. F. Accurate Thermochemistry from Quantum Chemical Calculations? Monatsh. Chem. 2008, 139, 309.

(18) Bowman, J. M.; Christoffel, K. M.; Tobin, F. Application of SCF-SI Theory to Vibrational Motion in Polyatomic Molecules. J. Phys. Chem. 1979, 83, 905.

(19) Christoffel, K. M.; Bowman, J. M. Application of SCF-SI Theory to Vibrational Motion in Polyatomic Molecules. Chem. Phys. Lett. 1982, $85,220$.

(20) Carter, S.; Bowman, J. M.; Handy, N. C. Extensions and Tests of "multimode": a Code to Obtain Accurate Vibration/rotation Energies of Many-mode Molecules. Theor. Chim. Acta 1998, 100, 191.

(21) Huang, X.; Braams, B. J.; Bowman, J. M. Ab Initio Potential Energy and Dipole Moment Surfaces of (H2O)2. J. Phys. Chem. A 2005, 110, 445.

(22) Teixeira, F.; Melo, A.; Cordeiro, M. N. D. S. Calibration sets and the accuracy of vibrational scaling factors: A case study with the X3LYP hybrid functional. J. Chem. Phys. 2010, 133, 114109.
(23) Pernot, P.; Cailliez, F. Comment on "Uncertainties in scaling factors for ab initio vibrational zero-point energies" [J. Chem. Phys. 130, 114102 (2009)] and "Calibration sets and the accuracy of vibrational scaling factors: A case study with the X3LYP hybrid functional" [J. Chem. Phys. 133, 114109 (2010)]. J. Chem. Phys. 2011, 134, 167101.

(24) Irikura, K. K.; Johnson, R. D.; Kacker, R. N.; Kessel, R. Response to "Comment on 'Uncertainties in scaling factors for ab initio vibrational zero-point energies' and 'Calibration sets and the accuracy of vibrational scaling factors: A case study with the X3LYP hybrid functional" J. Chem. Phys. 134, 167101 (2011). J. Chem. Phys. 2011, 134.

(25) Teixeira, F.; Melo, A.; Natalia, M.; Cordeiro, D. S. Response to "Comment on 'Uncertainties in scaling factors for ab initio vibrational zero-point energies' and 'Calibration sets and the accuracy of vibrational scaling factors: A case study with the X3LYP hybrid functional'”J. Chem. Phys. 134, 167101 (2011). J. Chem. Phys. 2011, 134.

(26) Borowski, P.; Fernandez-Gomez, M.; Fernandez-Liencres, M.; Ruiz, T. P. An Effective Scaling Frequency Factor Method for Scaling of Harmonic Vibrational Frequencies: Theory and Preliminary Application to Toluene. Chem. Phys. Lett. 2007, 446, 191.

(27) Borowski, P.; Drzewiecka, A.; Fernandez-Gomez, M.; FernandezLiencres, M.; Ruiz, T. P. An Effective Scaling Frequency Factor Method for Harmonic Vibrational Frequencies: The Factors' Transferability Problem. Chem. Phys. Lett. 2008, 465, 290.

(28) Borowski, P.; Drzewiecka, A.; Fernandez-Gomez, M.; FerandezLiencres, M. P.; Ruiz, T. P. A new, reduced set of scaling factors for both SQM and ESFF calculations. Vib. Spectrosc. 2010, 52, 16.

(29) Rauhut, G.; Pulay, P. Transferable Scaling Factors for Density Functional Derived Vibrational Force Fields. J. Phys. Chem. 1995, 99, 3093.

(30) Wolfs, I.; Desseyn, H. O. Modelling the vibrational behaviour of the cyclic carboxylic acid dimer. SQM force field of the formic acid dimer. J. Mol. Struct. (Theochem) 1996, 360, 81.

(31) Fabri, C.; Szidarovszky, T.; Magyarfalvi, G.; Tarczay, G. GasPhase and Ar-Matrix SQM Scaling Factors for Various DFT Functionals with Basis Sets Including Polarization and Diffuse Functions. J. Phys. Chem. A 2011, 115, 4640.

(32) Fernandez, L. E.; Marigliano, A. C. G.; Varetti, E. L. The vibrational properties of formic acid as monomer and dimer: a DFT study. Vib. Spectrosc. 2005, 37, 179.

(33) Kocevski, V.; Pejov, L. On the Assessment of Some New MetaHybrid and Generalized Gradient Approximation Functionals for Calculations of Anharmonic Vibrational Frequency Shifts in HydrogenBonded Dimers. J. Phys. Chem. A 2010, 114, 4354.

(34) Karthikeyan, S.; Singh, J. N.; Park, M.; Kumar, R.; Kim, K. S. Structures, Energetics, Vibrational Spectra of NH4+(H2O) $(n=4,6)$ Clusters: Ab Initio Calculations and First Principles Molecular Dynamics Simulations. J. Chem. Phys. 2008, 128, 244304.

(35) Pickard, F. C.; Pokon, E. K.; Liptak, M. D.; Shields, G. C. Comparison of CBS-QB3, CBS-APNO, G2, and G3 Thermochemical Predictions with Experiment for Formation of Ionic Clusters of Hydronium and Hydroxide Ions Complexed with Water. J. Chem. Phys. 2005, $122,024302$.

(36) Lee, H. M.; Suh, S. B.; Lee, J. Y.; Tarakeshwar, P.; Kim, K. S. Structures, Energies, Vibrational Spectra, and Electronic Properties of Water Monomer to Decamer. J. Chem. Phys. 2000, 112, 9759.

(37) Dunn, M. E.; Evans, T. M.; Kirschner, K. N.; Shields, G. C. Prediction of Accurate Anharmonic Experimental Vibrational Frequencies for Water Clusters, $(\mathrm{H} 2 \mathrm{O})(\mathrm{n}), \mathrm{n}=2-5$. J. Phys. Chem. A 2006, 110, 303.

(38) Losada, M.; Leutwlyer, S. Water Hexamer Clusters: Structures, Energies, and Predicted Mid-infrared Spectra. J. Chem. Phys. 2002, 117, 2003.

(39) Braly, L. B.; Liu, K.; Brown, M. G.; Keutsch, F. N.; Fellers, R. S.; Saykally, R. J. Terahertz Laser Spectroscopy of the Water Dimer Intermolecular Vibrations. II. (H2O)(2). J. Chem. Phys. 2000, 112, 10314.

(40) Ceponkus, J.; Uvdal, P.; Nelander, B. Intermolecular Vibrations of Different Isotopologs of the Water Dimer: Experiments and Density Functional Theory Calculations. J. Chem. Phys. 2008, 129, 194306. 
(41) Ceponkus, J.; Uvdal, P.; Nelander, B. Far-Infrared Band Strengths in the Water Dimer: Experiments and Calculations. J. Phys. Chem. A 2008, 112, 3921.

(42) Barone, V. Vibrational Zero-point Energies and Thermodynamic Functions Beyond the Harmonic Approximation. J. Chem. Phys. 2004, 120, 3059.

(43) Barone, V. Anharmonic Vibrational Properties by a Fully Automated Second-order Perturbative Approach. J. Chem. Phys. 2005, $122,014108$.

(44) Bowman, J. M. The Self-consistent-field Approach to Polyatomic Vibrations. Acc. Chem. Res. 1986, 19, 202.

(45) Gerber, R. B.; Ratner, M. A. A Semiclassical Self-consistent Field (SC SCF) Approximation for Eigenvalues of Coupled-Vibration Systems. Chem. Phys. Lett. 1979, 68, 195.

(46) Ratner, M. A.; Gerber, R. B. Excited Vibrational States of Polyatomic Molecules: the Semiclassical Self-consistent Field Approach. J. Phys. Chem. 1986, 90, 20.

(47) Xantheas, S. S. Anharmonic Vibrational Spectra of Hydrogen Bonded Clusters: Comparison between Higher Energy Derivative and Mean-field Grid Based Methods. Int. Rev. Phys. Chem. 2006, 25, 719.

(48) Jung, J.; Gerber, R. B. Vibrational Wave Functions and Spectroscopy of $(\mathrm{H} 2 \mathrm{O}) \mathrm{n}, \mathrm{n}=2$, 3, 4, 5: Vibrational Self-consistent Field with Correlation Corrections. J. Chem. Phys. 1996, 105, 10332.

(49) Chaban, G. M.; Jung, J. O.; Gerber, R. B. Anharmonic Vibrational Spectroscopy of Hydrogen-Bonded Systems Directly Computed from ab Initio Potential Surfaces: $(\mathrm{H} 2 \mathrm{O}) \mathrm{n}, \mathrm{n}=2$, 3; Cl- $(\mathrm{H} 2 \mathrm{O}) \mathrm{n}, \mathrm{n}=1,2$; $\mathrm{H}+(\mathrm{H} 2 \mathrm{O}) \mathrm{n}, \mathrm{n}=1$, 2; H2O-CH3OH. J. Phys. Chem. A 2000, 104, 2772.

(50) Njegic, B.; Gordon, M. S. Exploring the Effect of Anharmonicity of Molecular Vibrations on Thermodynamic Properties. J. Chem. Phys. 2006, 125, 224102.

(51) Child, M. S.; Lawton, R. T. Local and Normal Vibrational States: A Harmonically Coupled Anharmonic-oscillator Model. Faraday Discuss. Chem. Soc. 1981, 71, 273.

(52) Lee, M. S.; Baletto, F.; Kanhere, D. G.; Scandolo, S. Far-infrared Absorption of Water Clusters by First-principles Molecular Dynamics. J. Chem. Phys. 2008, 128, 214506.

(53) Begue, D.; Baraille, I.; Garrain, P. A.; Dargelos, A.; Tassaing, T. Calculation of IR Frequencies and Intensities in Electrical and Mechanical Anharmonicity Approximations: Application to Small Water Clusters. J. Chem. Phys. 2010, 133, 034102.

(54) Huang, X.; Braams, B. J.; Bowman, J. M.; Kelly, R. E. A.; Tennyson, J.; Groenenboom, G. C.; van der Avoird, A. New Ab initio Potential Energy Surface and the Vibration-rotation-tunneling Levels of (H2O) 2 and (D2O)2. J. Chem. Phys. 2008, 128, 034312.

(55) Su, J. T.; Xu, X.; Goddard, W. A. Accurate Energies and Structures for Large Water Clusters using the X3LYP Hybrid Density Functional. J. Phys. Chem. A 2004, 108, 10518.

(56) Ohno, K.; Okimura, M.; Akai, N.; Katsumoto, Y. The Effect of Cooperative Hydrogen Bonding on the $\mathrm{OH}$ Stretching-band Shift for Water Clusters Studied by Matrix-isolation Infrared Spectroscopy and Density Functional Theory. Phys. Chem. Chem. Phys. 2005, 7, 3005.

(57) Kjaergaard, H. G.; Garden, A. L.; Chaban, G. M.; Gerber, R. B.; Matthews, D. A.; Stanton, J. F. Calculation of Vibrational Transition Frequencies and Intensities in Water Dimer: Comparison of Different Vibrational Approaches. J. Phys. Chem. A 2008, 112, 4324.

(58) Diri, K.; Myshakin, E. M.; Jordan, K. D. On the Contribution of Vibrational Anharmonicity to the Binding Energies of Water Clusters. J Phys. Chem. A 2005, 109, 4005.

(59) Torrent-Sucarrat, M.; Anglada, J. M.; Luis, J. M. Role of Vibrational Anharmonicity in Atmospheric Radical Hydrogen-bonded Complexes. Phys. Chem. Chem. Phys. 2009, 11, 6377.

(60) Watanabe, Y.; Maeda, S.; Ohno, K. Intramolecular Vibrational Frequencies of Water Clusters $(\mathrm{H} 2 \mathrm{O})(\mathrm{n})(\mathrm{n}=2-5)$ : Anharmonic Analyses using Potential Functions Based on the Scaled Hypersphere Search Method. J. Chem. Phys. 2008, 129, 074315.

(61) Dykstra, C. E.; Shuler, K.; Young, R. A.; Bacic, Z. Anharmonicity Effects on Zero Point Energies of Weakly Bound Molecular Clusters. J. Mol. Struct. (Theochem) 2002, 591, 11.
(62) Isayev, O.; Gorb, L.; Leszczynski, J. Theoretical Calculations: Can Gibbs Free Energy for Intermolecular Complexes be Predicted Efficiently and Accurately? J. Comput. Chem. 2007, 28, 1598.

(63) Carbonniere, P.; Barone, V. Performances of Different Density Functionals in the Computation of Vibrational Spectra Beyond the Harmonic Approximation. Chem. Phys. Lett. 2004, 399, 226.

(64) Biczysko, M.; Panek, P.; Scalmani, G.; Bloino, J.; Barone, V. Harmonic and Anharmonic Vibrational Frequency Calculations with the Double-Hybrid B2PLYP Method: Analytic Second Derivatives and Benchmark Studies. J. Chem. Theory Comput. 2010, 6, 2115.

(65) Xantheas, S.; Dunning, T. Ab-Initio Studies Of Cyclic Water Clusters $(\mathrm{H} 2 \mathrm{o}) \mathrm{N}, \mathrm{N}=1-6$ 0.1. Optimal Structures And VibrationalSpectra. J. Chem. Phys. 1993, 99, 8774.

(66) Xantheas, S. S. Ab initio Studies of Cyclic Water Clusters $(\mathrm{H} 2 \mathrm{O}) \mathrm{n}, \mathrm{n}=1-6$. II. Analysis of Many-body Interactions. J. Chem. Phys. 1994, 100, 7523.

(67) Xantheas, S. S. Ab initio Studies of Cyclic Water Clusters $(\mathrm{H} 2 \mathrm{O}) \mathrm{n}, \mathrm{n}=1-6$. III. Comparison of Density Functional with MP2 Results. J. Chem. Phys. 1995, 102, 4505.

(68) Xantheas, S. S. On the Importance of the Fragment Relaxation Energy Terms in the Estimation of the Basis Set Superposition Error Correction to the Intermolecular Interaction Energy. J. Chem. Phys. 1996, 104, 8821.

(69) Xantheas, S. S. Significance of Higher-order Many-body Interaction Energy Terms in Water Clusters and Bulk Water. Philos. Mag. B 1996, 73, 107.

(70) Xantheas, S. S. Cooperativity and Hydrogen Bonding Network in Water Clusters. Chem. Phys. 2000, 258, 225.

(71) Burnham, C. J.; Xantheas, S. S. Development of Transferable Interaction Models for Water. IV. A Flexible, All-atom Polarizable Potential (TTM2-F) Based on Geometry Dependent Charges Derived from an Ab initio Monomer Dipole Moment Surface. J. Chem. Phys. 2002, 116, 5115.

(72) Burnham, C. J.; Xantheas, S. S. Development of Transferable Interaction Models for Water. I. Prominent Features of the Water Dimer Potential Energy Surface. J. Chem. Phys. 2002, 116, 1479.

(73) Burnham, C. J.; Xantheas, S. S. Development of Transferable Interaction Models for Water. III. Reparametrization of an All-atom Polarizable Rigid Model (TTM2-R) from First Principles. J. Chem. Phys. 2002, 116, 1500.

(74) Burnham, C. J.; Xantheas, S. S.; Miller, M. A.; Applegate, B. E.; Miller, R. E. The Formation of Cyclic Water Complexes by Sequential Ring Insertion: Experiment and Theory. J. Chem. Phys. 2002, 117, 1109.

(75) Xantheas, S. S.; Burnham, C. J.; Harrison, R. J. Development of Transferable Interaction Models for Water. II. Accurate Energetics of the First Few Water Clusters from First Principles. J. Chem. Phys. 2002, 116,1493

(76) Bulusu, S.; Yoo, S.; Apra, E.; Xantheas, S.; Zeng, X. C. Lowestenergy structures of water clusters $(\mathrm{H} 2 \mathrm{O})(11)$ and $(\mathrm{H} 2 \mathrm{O})(13)$. J Phys. Chem. A 2006, 110, 11781.

(77) Xantheas, S. S.; Apra, E. The Binding Energies of the D2d and S4 Water Octamer Isomers: High-level Electronic Structure and Empirical Potential Results. J. Chem. Phys. 2004, 120, 823.

(78) Xantheas, S. S. Interaction Potentials for Water from Accurate Cluster Calculations. In Intermolecular Forces and Clusters II; Pacific Northwest National Laboratory: Richland, WA, 2005; Vol. 116; p 119.

(79) Yoo, S.; Kirov, M. V.; Xantheas, S. S. Low-Energy Networks of the T-Cage $(\mathrm{H} 2 \mathrm{O})(24)$ Cluster and Their Use in Constructing Periodic Unit Cells of the Structure I (sl) Hydrate Lattice. J. Am. Chem. Soc. 2009, 131,7564

(80) Yoo, S.; Apra, E.; Zeng, X. C.; Xantheas, S. S. High-Level Ab Initio Electronic Structure Calculations of Water Clusters (H2O) 16 and (H2O)17: A New Global Minimum for (H2O)16. J. Phys. Chem. Lett. 2010, 1, 3122.

(81) Isaacson, A. D. Removing Resonance Effects from Quantum Mechanical Vibrational Partition Functions Obtained from Perturbation Theory. J. Chem. Phys. 1998, 108, 9978.

(82) Frisch, M. J.; Trucks, G. W.; Schlegel, H. B.; Scuseria, G. E.; Robb, M. A.; Cheeseman, J. R.; Montgomery, J. A.; Vreven, T.; 
Kudin, K. N.; Burant, J. C.; Millam, J. M.; Iyengar, S. S.; Tomasi, J.; Barone, V.; Mennucci, B.; Cossi, M.; Scalmani, G.; Rega, N.; Petersson, G. A.; Nakatsuji, H.; Hada, M.; Ehara, M.; Toyota, K.; Fukuda, R.; Hasegawa, J.; Ishida, M.; Nakajima, T.; Honda, Y.; Kitao, O.; Nakai, H.; Klene, M.; Li, X.; Knox, J. E.; Hratchian, H. P.; Cross, J. B.; Bakken, V.; Adamo, C.; Jaramillo, J.; Gomperts, R.; Stratmann, R. E.; Yazyev, O.; Austin, A. J.; Cammi, R.; Pomelli, C.; Ochterski, J. W.; Ayala, P. Y.; Morokuma, K.; Voth, G. A.; Salvador, P.; Dannenberg, J. J.; Zakrzewski, V. G.; Dapprich, S.; Daniels, A. D.; Strain, M. C.; Farkas, O.; Malick, D. K.; Rabuck, A. D.; Raghavachari, K.; Foresman, J. B.; Ortiz, J. V.; Cui, Q.; Baboul, A. G.; Clifford, S.; Cioslowski, J.; Stefanov, B. B.; Liu, G.; Liashenko, A.; Piskorz, P.; Komaromi, I.; Martin, R. L.; Fox, D. J.; Keith, T.; Al-Laham, M. A.; Peng, C. Y.; Nanayakkara, A.; Challacombe, M.; Gill, P. M. W.; Johnson, B.; Chen, W.; Wong, M. W.; Gonzalez, C.; Pople, J. A. Gaussian 09, revision A.02; Gaussian, Inc.: Wallingford, CT, 2009.

(83) Martin, J. M. L.; Lee, T. J.; Taylor, P. R.; Francois, J. P. The Anharmonic Force Field of Ethylene, $\mathrm{C} 2 \mathrm{H} 4$ by Means of Accurate $\mathrm{Ab}$ initio Calculations. J. Chem. Phys. 1995, 103, 2589.

(84) Boese, A. D.; Martin, J. M. L. Vibrational Spectra of the Azabenzenes Revisited: Anharmonic Force Fields. J. Phys. Chem. A 2004, 108, 3085.

(85) Frisch, M. J.; Trucks, G. W.; Schlegel, H. B.; Scuseria, G. E.; Robb, M. A.; Cheeseman, J. R.; Montgomery, J. A.; Vreven, T.; Kudin, K. N.; Burant, J. C.; Millam, J. M.; Iyengar, S. S.; Tomasi, J.; Barone, V.; Mennucci, B.; Cossi, M.; Scalmani, G.; Rega, N.; Petersson, G. A.; Nakatsuji, H.; Hada, M.; Ehara, M.; Toyota, K.; Fukuda, R.; Hasegawa, J.; Ishida, M.; Nakajima, T.; Honda, Y.; Kitao, O.; Nakai, H.; Klene, M.; Li, X.; Knox, J. E.; Hratchian, H. P.; Cross, J. B.; Bakken, V.; Adamo, C.; Jaramillo, J.; Gomperts, R.; Stratmann, R. E.; Yazyev, O.; Austin, A. J.; Cammi, R.; Pomelli, C.; Ochterski, J. W.; Ayala, P. Y.; Morokuma, K.; Voth, G. A.; Salvador, P.; Dannenberg, J. J.; Zakrzewski, V. G.; Dapprich, S.; Daniels, A. D.; Strain, M. C.; Farkas, O.; Malick, D. K.; Rabuck, A. D.; Raghavachari, K.; Foresman, J. B.; Ortiz, J. V.; Cui, Q.; Baboul, A. G.; Clifford, S.; Cioslowski, J.; Stefanov, B. B.; Liu, G.; Liashenko, A.; Piskorz, P.; Komaromi, I.; Martin, R. L.; Fox, D. J.; Keith, T.; AlLaham, M. A.; Peng, C. Y.; Nanayakkara, A.; Challacombe, M.; Gill, P. M. W.; Johnson, B.; Chen, W.; Wong, M. W.; Gonzalez, C.; Pople, J. A. Gaussian 03, revisions D.01 and E.01); Gaussian, Inc.: Wallingford, CT, 2004.

(86) Stanton, J. F.; Gauss, J.; Harding, M. E.; Szalay, P. G.; Auer, A. A.; Bartlett, R. J.; Benedikt, U.; Berger, C.; Bernholdt, D. E.; Bomble, Y. J.; Christiansen, O.; Heckert, M.; Heun, O.; Huber, C.; Jagau, T. -C.; Jonsson, D.; J. Jusèlius; Klein, K.; Lauderdale, W. J.; Matthews, D.; Metzroth, T.; D. P. O’Neill; Price, D. R.; Prochnow, E.; Ruud, K.; Schiffmann, F.; Stopkowicz, S.; Varner, M. E.; J. Vázquez; Watts, J. D.; Wang, F.; Almlöf, J.; Taylor, P. R.; Helgaker, T.; Jørgensen, P.; Jensen, H. J. A.; Olsen, J.; Mitin, A. V.; Wüllen, C. v. CFOUR (Coupled Cluster Techniques for Computational Chemistry), a quantum-chemical program package; The University of Texas at Austin, Johannes GutenbergUniversität Mainz, and Eötvös-Lorand-University Budapest: Austin, TX, Mainz, Germany, and Budapest, Hungary, 2010.

(87) Csonka, G. I.; Ruzsinszky, A.; Perdew, J. P. Estimation, Computation, and Experimental Correction of Molecular Zero-point Vibrational Energies. J Phys. Chem. A 2005, 109, 6779.

(88) McQuarrie, D. A. Statistical Thermodynamics; Harper \& Row: New York, 1973.

(89) Truhlar, D. G.; Isaacson, A. D. Simple Perturbation Theory Estimates of Equilibrium Constants from Force Fields. J. Chem. Phys. 1991, 94, 357.

(90) Isaacson, A. D. Anharmonic Effects on the Transition State Theory Rate Constant. J. Chem. Phys. 2002, 117, 8778.

(91) Isaacson, A. D. Including Anharmonicity in the Calculation of Rate Constants. 1. The HCN/HNC Isomerization Reaction. J. Phys. Chem. A 2006, 110, 379.

(92) Kurten, T.; Noppel, M.; Vehkamaki, H.; Salonen, M.; Kulmala, M. Quantum Chemical Studies of Hydrate Formation of $\mathrm{H} 2 \mathrm{SO} 4$ and HSO4. Boreal Environ. Res. 2007, 12, 431.
(93) Dunn, M. E.; Pokon, E. K.; Shields, G. C. Thermodynamics of Forming Water Clusters at Various Temperatures and Pressures by Gaussian-2, Gaussian-3, Complete Basis Set-QB3, and Complete Basis Set-APNO Model Chemistries; Implications for Atmospheric Chemistry. J. Am. Chem. Soc. 2004, 126, 2647.

(94) Morrell, T. E.; Shields, G. C. Atmospheric Implications for Formation of Clusters of Ammonium and 1-10 Water Molecules. J. Phys. Chem. A 2010, 114, 4266. 


\title{
Supporting Information for:
}

\section{The Role of Anharmonicity in Hydrogen Bonded Systems: The Case of Water Clusters}

\author{
Berhane Temelso and George C. Shields ${ }^{*}$ \\ Dean's Office, College of Arts and Sciences, and \\ Department of Chemistry \\ Bucknell University \\ Lewisburg, PA 17837
}

July 11, 2011

*Correspondence: george.shields@bucknell.edu 
Table S1: $\left(\mathrm{H}_{2} \mathrm{O}\right)_{2}$-Cs geometry, harmonic and VPT2 fundamental frequencies

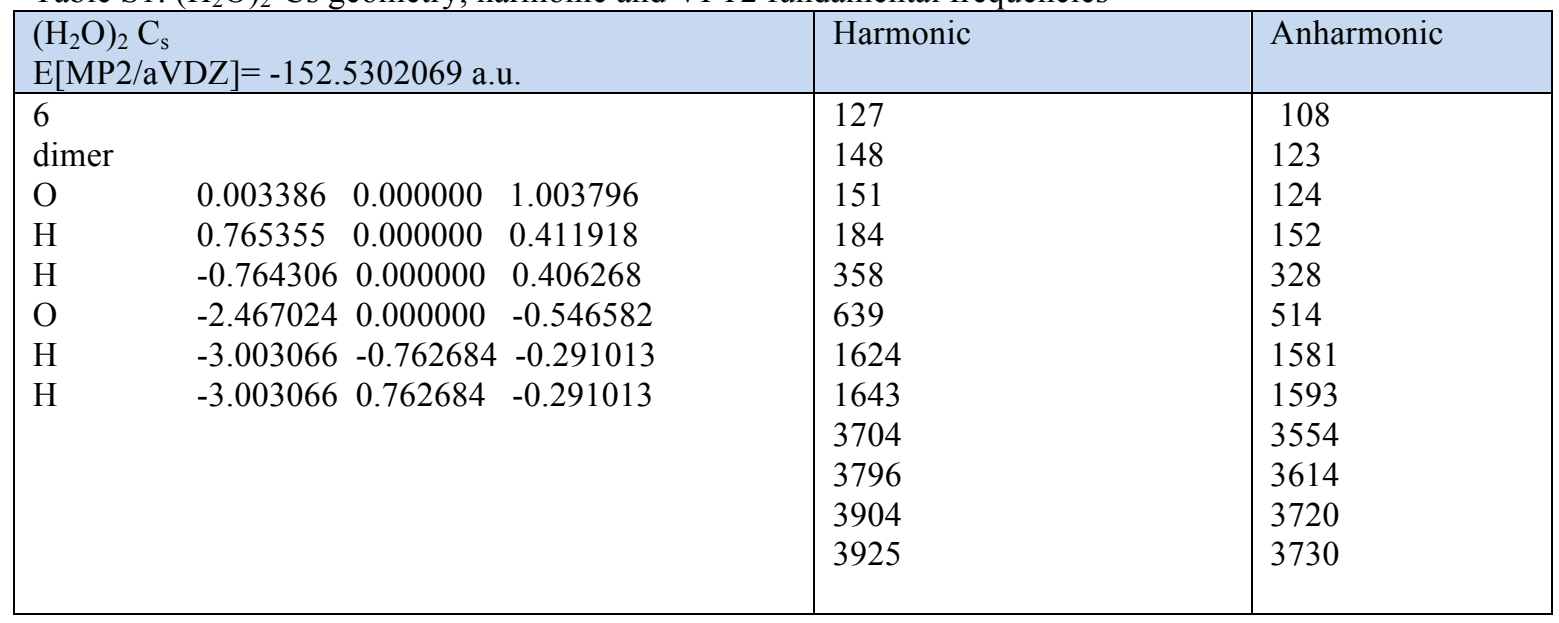

Table S2: $\left(\mathrm{H}_{2} \mathrm{O}\right)_{3}$-UUD geometry, harmonic and VPT2 fundamental frequencies

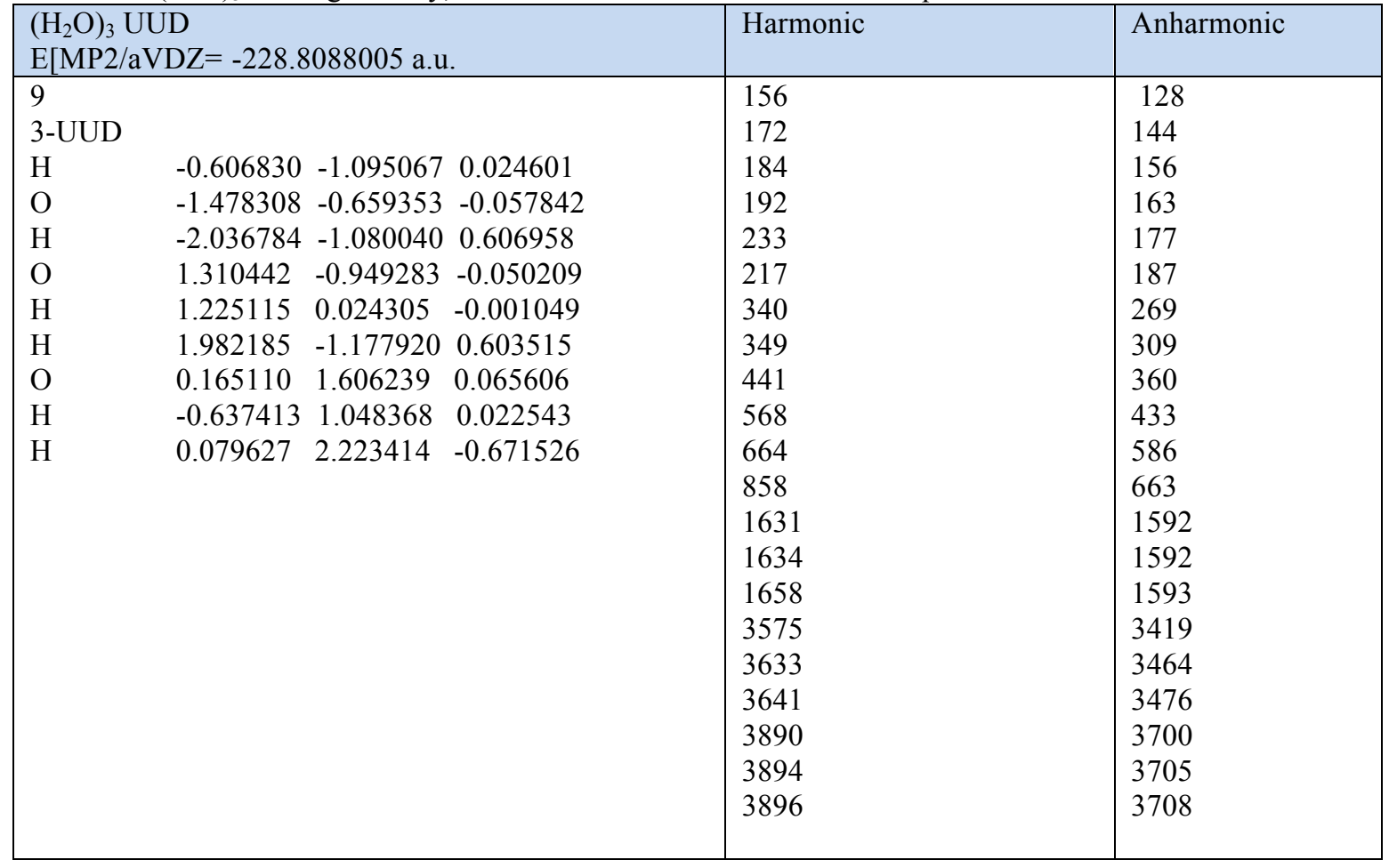


Table S3: $\left(\mathrm{H}_{2} \mathrm{O}\right)_{4}$-S4 geometry, harmonic and VPT2 fundamental frequencies

\begin{tabular}{|c|c|c|c|c|c|}
\hline \multicolumn{4}{|c|}{$\begin{array}{l}\left(\mathrm{H}_{2} \mathrm{O}\right)_{4} \mathrm{~S}_{4} \\
\mathrm{E}[\mathrm{MP} 2 / \mathrm{aVDZ}]=-305.0894329 \text { a.u. }\end{array}$} & Harmonic & Anharmonic \\
\hline \multirow{2}{*}{\multicolumn{4}{|c|}{$\begin{array}{l}12 \\
4-S 4\end{array}$}} & 49.958 & 45.75 \\
\hline & & & & 78.917 & 70.52 \\
\hline $\mathrm{H}$ & 1.214527 & 2.216388 & 0.764022 & 209.193 & 185.25 \\
\hline $\mathrm{O}$ & 1.023132 & 1.651613 & 0.005269 & 199.363 & 182.71 \\
\hline $\mathrm{H}$ & 1.561716 & -0.042153 & 0.004066 & 235.054 & 202.32 \\
\hline $\mathrm{H}$ & 0.042152 & 1.561715 & -0.004066 & 235.054 & 202.32 \\
\hline $\mathrm{O}$ & 1.651615 & -1.023133 & -0.005269 & 252.417 & 221.88 \\
\hline $\mathrm{H}$ & 2.216390 & -1.214529 & -0.764021 & 252.417 & 221.89 \\
\hline $\mathrm{H}$ & -0.042154 & -1.561717 & -0.004066 & 259.03 & 231.64 \\
\hline $\mathrm{O}$ & -1.023134 & -1.651612 & 0.005269 & 288.225 & 252.95 \\
\hline $\mathrm{H}$ & -1.214531 & -2.216387 & 0.764021 & 400.093 & 346.13 \\
\hline $\mathrm{H}$ & -1.561714 & 0.042152 & 0.004066 & 431.342 & 384.29 \\
\hline $\mathrm{O}$ & -1.651612 & 1.023132 & -0.005269 & 447.982 & 398.04 \\
\hline \multirow[t]{17}{*}{$\mathrm{H}$} & -2.216387 & 1.214529 & -0.764021 & 447.982 & 398.05 \\
\hline & & & & 748.337 & 694.58 \\
\hline & & & & 819.328 & 718.26 \\
\hline & & & & 819.329 & 718.27 \\
\hline & & & & 989.556 & 837.26 \\
\hline & & & & 1636.002 & 1595.31 \\
\hline & & & & 1651.185 & 1616.18 \\
\hline & & & & 1651.185 & 1616.18 \\
\hline & & & & 1681.262 & 1658.55 \\
\hline & & & & 3395.65 & 3249.86 \\
\hline & & & & 3486.321 & 3316.26 \\
\hline & & & & 3486.321 & 3316.28 \\
\hline & & & & 3523.551 & 3349.67 \\
\hline & & & & 3884.226 & 3692.17 \\
\hline & & & & 3885.528 & 3694.36 \\
\hline & & & & 3884.929 & 3694.37 \\
\hline & & & & 3884.929 & 3693.04 \\
\hline
\end{tabular}


Table S4: $\left(\mathrm{H}_{2} \mathrm{O}\right)_{4}-\mathrm{C}_{\mathrm{i}}$ geometry, harmonic and VPT2 fundamental frequencies

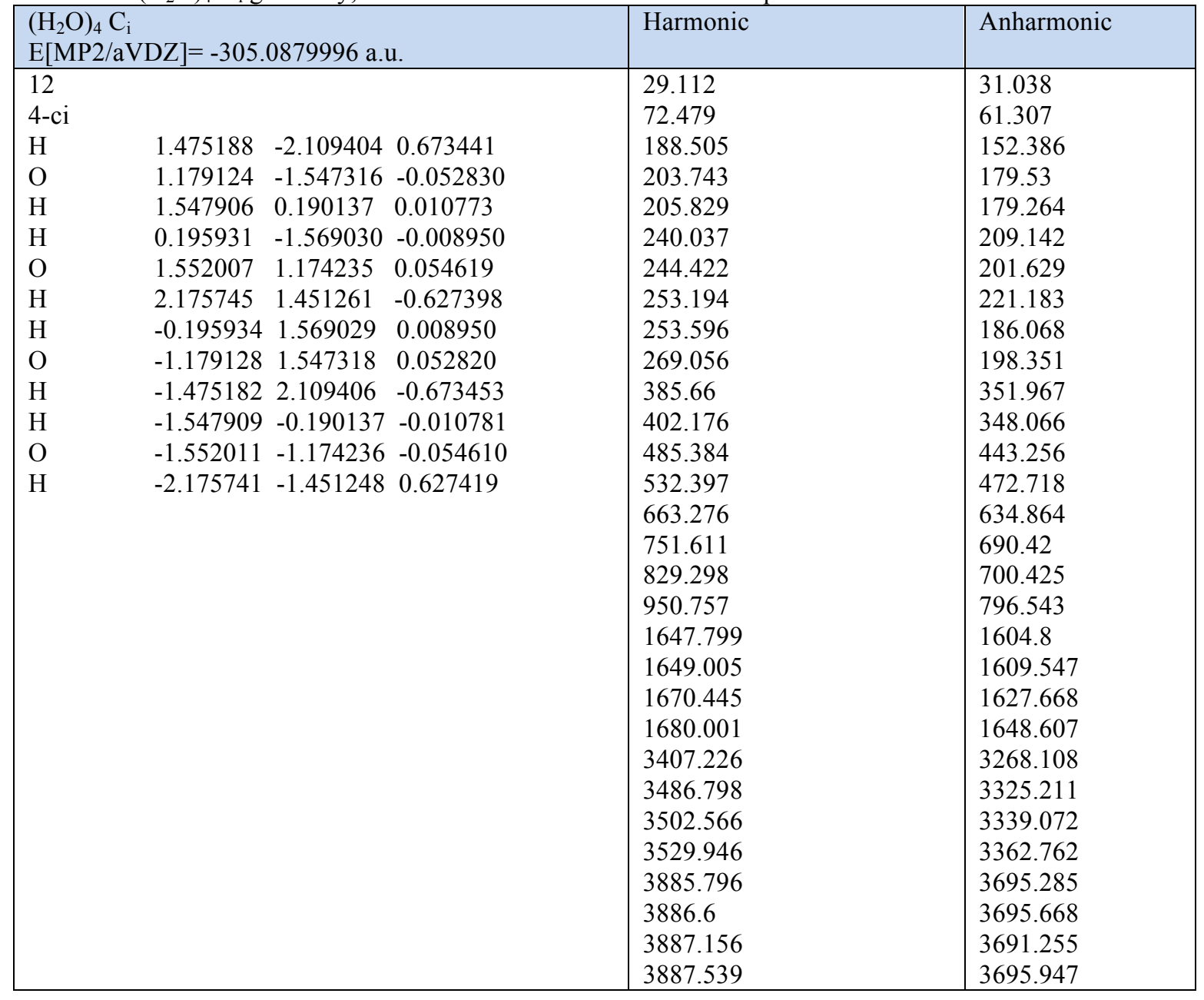


Table S5: $\left(\mathrm{H}_{2} \mathrm{O}\right)_{5}$-Cyclic geometry, harmonic and VPT2 fundamental frequencies

\begin{tabular}{|c|c|c|c|}
\hline \multicolumn{2}{|c|}{$\begin{array}{l}\left(\mathrm{H}_{2} \mathrm{O}\right)_{5} \text { Cyclic } \\
\mathrm{E}[\mathrm{MP} 2 / \mathrm{aVDZ}]=-381.3647344 \text { a.u. }\end{array}$} & Harmonic & Anharmonic \\
\hline \multirow{2}{*}{\multicolumn{2}{|c|}{$\begin{array}{l}15 \\
5 \text {-cyclic }\end{array}$}} & 23.483 & 19.545 \\
\hline & & 42.464 & 32.803 \\
\hline $\mathrm{H}$ & $\begin{array}{lll}-0.972759 & -1.684129 & -0.084067\end{array}$ & 62.567 & 49.666 \\
\hline $\mathrm{H}$ & $\begin{array}{lll}2.526700 & -1.272626 & 0.968055\end{array}$ & 65.39 & 43.205 \\
\hline $\mathrm{O}$ & $\begin{array}{lll}-0.204724 & -2.296294 & -0.177775\end{array}$ & 180.843 & 157.233 \\
\hline $\mathrm{O}$ & $\begin{array}{lll}-2.266352 & -0.528691 & 0.097773\end{array}$ & 193.115 & 137.635 \\
\hline $\mathrm{H}$ & $\begin{array}{llll}-1.938217 & 0.400557 & 0.073642\end{array}$ & 200.039 & 147.745 \\
\hline $\mathrm{H}$ & $\begin{array}{lll}-2.782296 & -0.590427 & 0.910681\end{array}$ & 228.527 & 169.05 \\
\hline $\mathrm{O}$ & $\begin{array}{lll}-1.185952 & 1.982012 & -0.081836\end{array}$ & 235.965 & 205.269 \\
\hline $\mathrm{H}$ & $-1.4001372 .719681 \quad 0.501615$ & 240.589 & 206.84 \\
\hline $\mathrm{H}$ & $\begin{array}{llll}-0.200693 & 1.928468 & -0.070905\end{array}$ & 266.553 & 214.871 \\
\hline $\mathrm{O}$ & $\begin{array}{lll}1.533013 & 1.731673 & -0.048325\end{array}$ & 295.769 & 248.8 \\
\hline $\mathrm{H}$ & $\begin{array}{lll}1.786163 & 0.783446 & 0.052976\end{array}$ & 299.557 & 260.721 \\
\hline $\mathrm{H}$ & $\begin{array}{lll}2.007636 & 2.025999 & -0.835448\end{array}$ & 302.504 & 266.5 \\
\hline $\mathrm{O}$ & $\begin{array}{llll}2.125877 & -0.922617 & 0.163277\end{array}$ & 410.579 & 375.47 \\
\hline $\mathrm{H}$ & $\begin{array}{lll}1.306860 & -1.457570 & 0.034316\end{array}$ & 429.896 & 375.877 \\
\hline & $\begin{array}{lll}-0.355647 & -2.755632 & -1.012842\end{array}$ & 453.163 & 400.484 \\
\hline & & 466.338 & 415.693 \\
\hline & & 512.63 & 468.686 \\
\hline & & 721.191 & 669.636 \\
\hline & & 787.07 & 716.823 \\
\hline & & 865.438 & 744.253 \\
\hline & & 881.125 & 769.378 \\
\hline & & 984.4 & 857.331 \\
\hline & & 1640.467 & 1598.058 \\
\hline & & 1652.082 & 1621.863 \\
\hline & & 1660.213 & 1636.922 \\
\hline & & 1681.013 & 1627.78 \\
\hline & & 1688.845 & 1602.13 \\
\hline & & 3353.788 & 3144.346 \\
\hline & & 3433.326 & 3275.333 \\
\hline & & 3442.183 & 3282.28 \\
\hline & & 3486.953 & 3313.942 \\
\hline & & 3494.005 & 3324.797 \\
\hline & & 3882.765 & 3689.378 \\
\hline & & 3884.968 & 3689.106 \\
\hline & & 3886.789 & 3694.497 \\
\hline & & 3886.981 & 3696.495 \\
\hline & & 3888.698 & 3699.29 \\
\hline
\end{tabular}


Table S6: $\left(\mathrm{H}_{2} \mathrm{O}\right)_{6}$-Cyclic chair geometry, harmonic and VPT2 fundamental frequencies

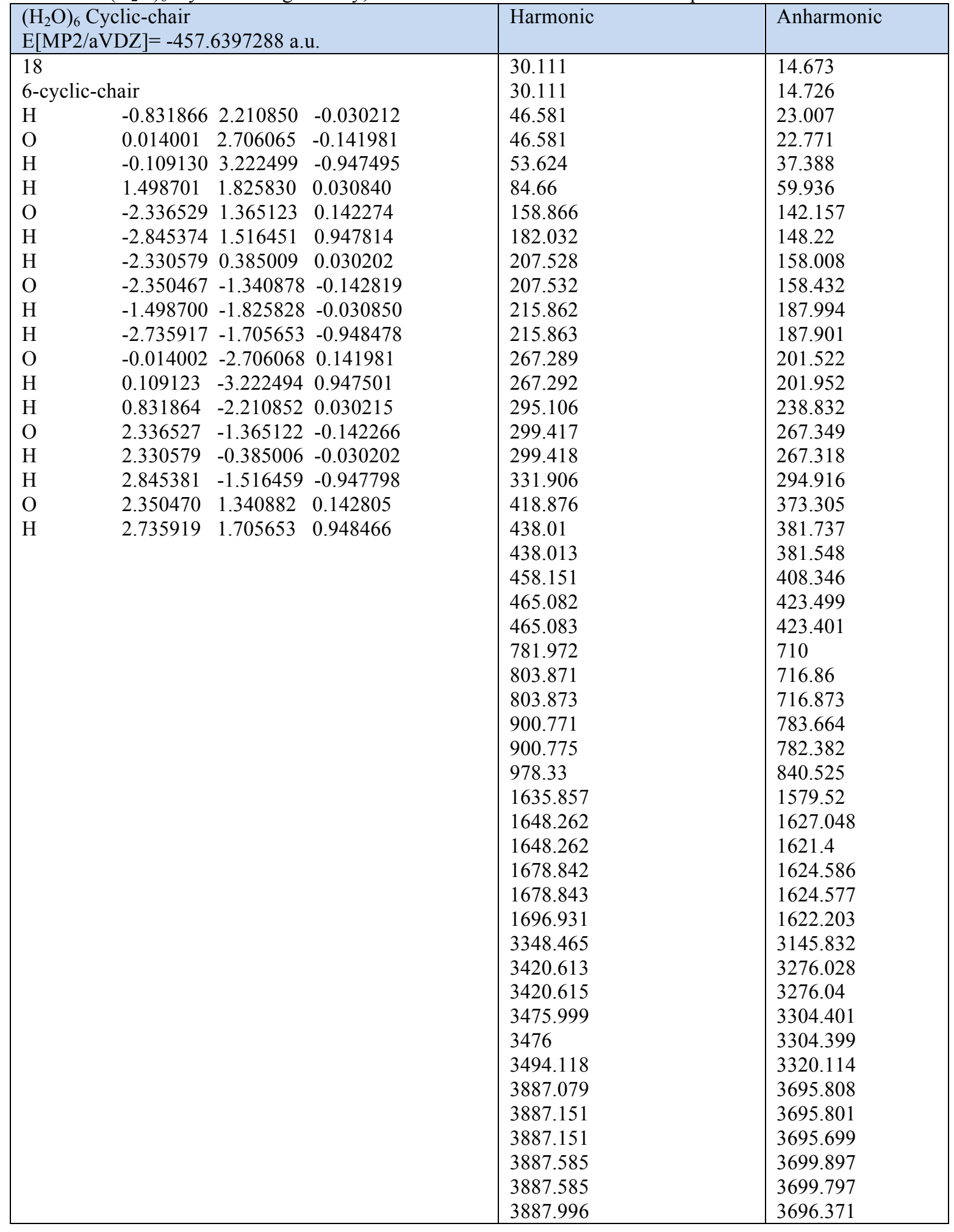


Table S7: $\left(\mathrm{H}_{2} \mathrm{O}\right)_{6}$-Book-1 geometry, harmonic and VPT2 fundamental frequencies

\begin{tabular}{|c|c|c|c|c|c|}
\hline \multicolumn{4}{|c|}{$\begin{array}{l}\left(\mathrm{H}_{2} \mathrm{O}\right)_{6} \text { Book-1 } \\
\mathrm{E}[\mathrm{MP} 2 / \mathrm{aVDZ}]=-457.6418357 \text { a.u. }\end{array}$} & \multirow{2}{*}{$\begin{array}{l}\text { Harmonic } \\
27.784\end{array}$} & Anharmonic \\
\hline \multirow{2}{*}{\multicolumn{4}{|c|}{$\begin{array}{l}18 \\
6 \text {-book-1 }\end{array}$}} & & 16.954 \\
\hline & & & & 38.786 & 29.743 \\
\hline \multicolumn{4}{|c|}{$\begin{array}{llll}\mathrm{H} & 3.587741 & 5.431675 & 4.279365\end{array}$} & 55.58 & 46.161 \\
\hline $\mathrm{H}$ & 4.099179 & 5.244158 & 5.738394 & 69.498 & 55.939 \\
\hline $\mathrm{H}$ & 6.184675 & 5.081186 & 4.706913 & 87.655 & 77.592 \\
\hline $\mathrm{H}$ & 7.554866 & 5.712418 & 4.979451 & 155.642 & 125.967 \\
\hline $\begin{array}{l}\mathrm{H} \\
\mathrm{H}\end{array}$ & 7.139008 & 7.896633 & 4.985358 & 182.029 & 158.487 \\
\hline $\mathrm{H}$ & 8.512417 & 7.867699 & 4.283524 & 189.464 & 157.992 \\
\hline $\mathrm{H}$ & 4.957618 & 8.810819 & 5.597089 & 198.218 & 172.251 \\
\hline $\mathrm{H}$ & 4.881584 & 7.418557 & 4.933387 & 227.824 & 198.432 \\
\hline $\mathrm{H}$ & 3.803815 & 4.808027 & 8.033109 & 236.666 & 206.61 \\
\hline $\mathrm{H}$ & 4.931421 & 4.005915 & 7.333809 & 254.05 & 208.726 \\
\hline $\mathrm{H}$ & 6.909855 & 4.031200 & 6.465899 & 255.276 & 215.672 \\
\hline $\mathrm{H}$ & 6.905003 & 2.684257 & 7.240083 & 279.262 & 247.545 \\
\hline $\mathrm{O}$ & 4.293859 & 5.700184 & 4.879957 & 289.398 & 247.165 \\
\hline $\mathrm{O}$ & 7.094090 & 4.847207 & 4.962753 & 297.991 & 264.35 \\
\hline $\mathrm{O}$ & 8.032034 & 7.491761 & 5.031418 & 311.099 & 270.55 \\
\hline $\mathrm{O}$ & 5.364980 & 8.271329 & 4.908550 & 387.276 & 347.533 \\
\hline $\mathrm{O}$ & 6.606979 & 3.599541 & 7.305362 & 403.125 & 357.057 \\
\hline $\mathrm{O}$ & 4.017277 & 4.363336 & 7.203678 & 442.628 & 410.865 \\
\hline & & & & 453.943 & 407.544 \\
\hline & & & & 480.956 & 437.979 \\
\hline & & & & 543.016 & 475.286 \\
\hline & & & & 616.587 & 521.773 \\
\hline & & & & 730.608 & 648.548 \\
\hline & & & & 754.782 & 666.495 \\
\hline & & & & 837.913 & 728.964 \\
\hline & & & & 858.437 & 753.348 \\
\hline & & & & 907.688 & 808.225 \\
\hline & & & & 1025.417 & 900.824 \\
\hline & & & & 1631.551 & 1614.821 \\
\hline & & & & 1644.984 & 1607.997 \\
\hline & & & & 1647.123 & 1614.034 \\
\hline & & & & 1666.651 & 1625.812 \\
\hline & & & & 1678.307 & 1635.642 \\
\hline & & & & 1707.409 & 1670.153 \\
\hline & & & & 3285.43 & 3068.04 \\
\hline & & & & 3362.287 & 3169.735 \\
\hline & & & & 3414.879 & 3265.397 \\
\hline & & & & 3515.719 & 3355.648 \\
\hline & & & & 3570.074 & 3397.803 \\
\hline & & & & 3586.175 & 3415.438 \\
\hline & & & & 3734.822 & 3546.317 \\
\hline & & & & 3877.443 & 3685.701 \\
\hline & & & & 3882.208 & 3691.765 \\
\hline & & & & 3884.865 & 3693.115 \\
\hline & & & & 3886.254 & 3693.712 \\
\hline & & & & 3888.919 & 3697.177 \\
\hline
\end{tabular}


Table S8: $\left(\mathrm{H}_{2} \mathrm{O}\right)_{6}$-Cage geometry, harmonic and VPT2 fundamental frequencies

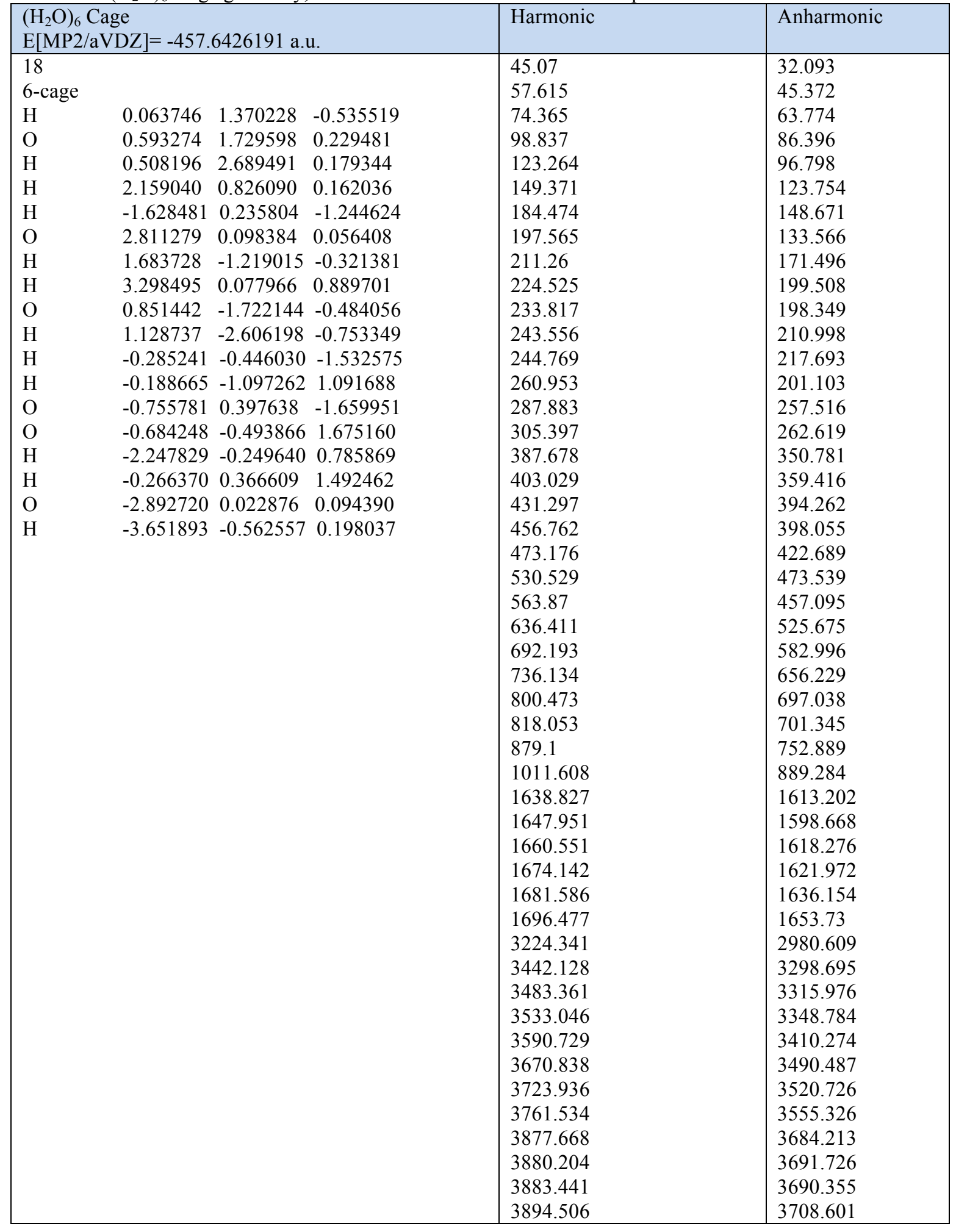


Table S9: $\left(\mathrm{H}_{2} \mathrm{O}\right)_{6}$-Prism geometry, harmonic and VPT2 fundamental frequencies

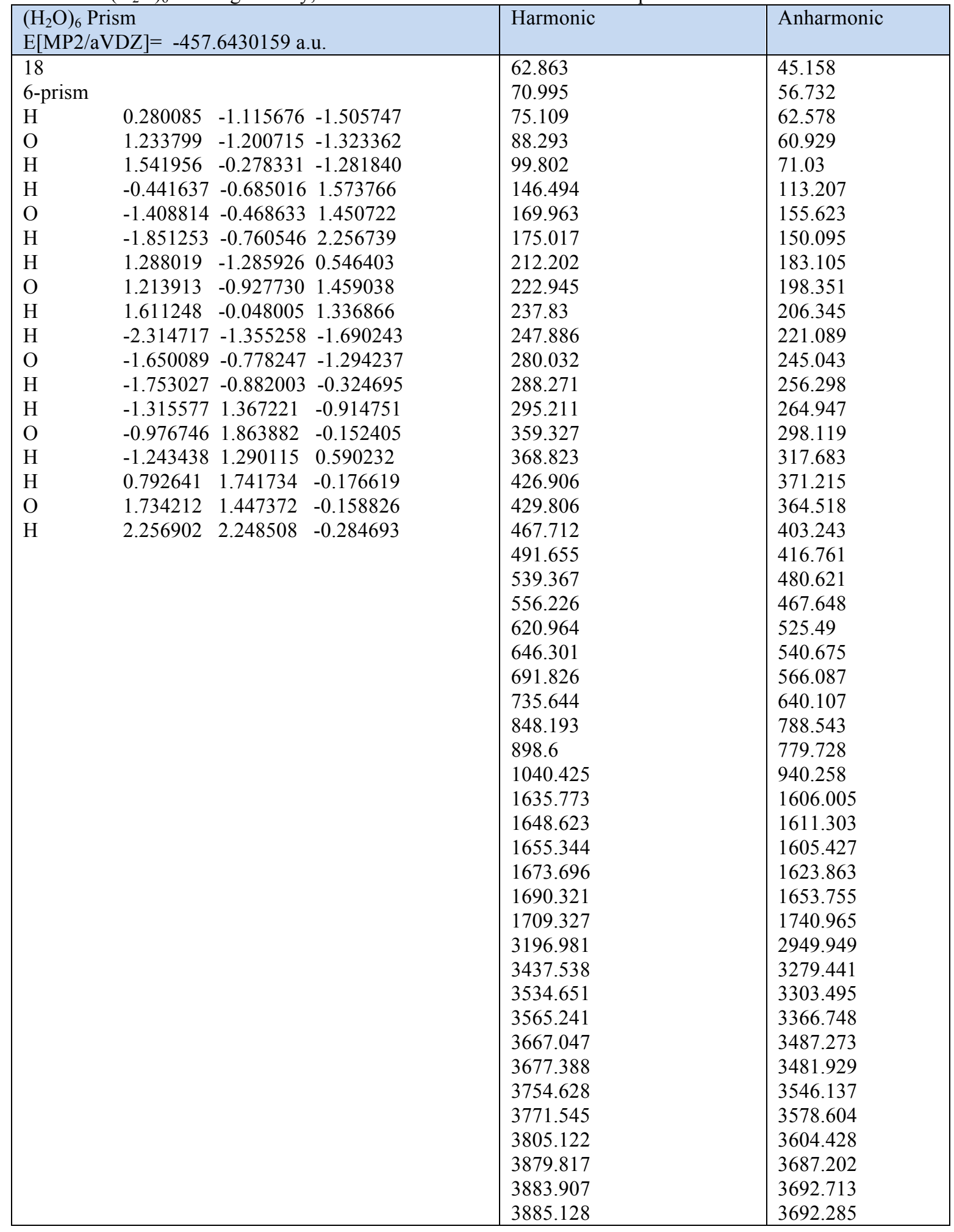


Table S10: $\left(\mathrm{H}_{2} \mathrm{O}\right)_{6}$-Book-2 geometry, harmonic and VPT2 fundamental frequencies

\begin{tabular}{|c|c|c|c|c|c|}
\hline \multicolumn{4}{|c|}{$\begin{array}{l}\left(\mathrm{H}_{2} \mathrm{O}\right)_{6} \text { Book-2 } \\
\text { E[MP2/aVDZ] }=-457.6414447 \text { a.u. }\end{array}$} & \multirow{2}{*}{$\begin{array}{l}\text { Harmonic } \\
23.148\end{array}$} & Anharmonic \\
\hline \multirow{2}{*}{\multicolumn{4}{|c|}{$\begin{array}{l}18 \\
6 \text {-book-2 }\end{array}$}} & & 2.588 \\
\hline & & & & 30.76 & 19.265 \\
\hline \multirow{2}{*}{\multicolumn{4}{|c|}{$\begin{array}{llll}\mathrm{O} & 2.305230 & 1.271784 & -0.403092 \\
\mathrm{H} & 3.223784 & 1.463814 & -0.178001\end{array}$}} & 40.5 & 37.999 \\
\hline & & & & 68.645 & 46.964 \\
\hline \multirow{2}{*}{$\begin{array}{l}\mathrm{H} \\
\mathrm{O}\end{array}$} & 2.286365 & 0.298477 & -0.587624 & 85.795 & 68.629 \\
\hline & 2.025571 & -1.399657 & -0.678701 & 153.552 & 123.61 \\
\hline $\mathrm{H}$ & 1.775147 & -1.804813 & -1.518038 & 190.23 & 165.967 \\
\hline $\mathrm{H}$ & 1.242649 & -1.534250 & -0.085401 & 205.171 & 178.941 \\
\hline c & -0.089941 & -1.445594 & 1.017708 & 210.101 & 182.116 \\
\hline $\mathrm{H}$ & -0.072179 & -0.521941 & 1.323776 & 223.618 & 199.601 \\
\hline $\mathrm{H}$ & -0.930735 & -1.500142 & 0.516594 & 237.071 & 207.952 \\
\hline $\mathrm{O}$ & -2.367388 & -1.241556 & -0.605451 & 251.524 & 221.758 \\
\hline $\mathrm{H}$ & -3.264516 & -1.467065 & -0.330597 & 261.723 & 225.28 \\
\hline $\mathrm{H}$ & -2.355823 & -0.260300 & -0.654295 & 269.755 & 230.683 \\
\hline $\mathrm{O}$ & -2.008184 & 1.505372 & -0.534949 & 285.671 & 251.81 \\
\hline $\mathrm{H}$ & -1.750468 & 1.979067 & -1.335618 & 290.529 & 261.119 \\
\hline $\mathrm{H}$ & -1.242086 & 1.602725 & 0.069499 & 298.742 & 238.775 \\
\hline $\mathrm{O}$ & 0.162802 & 1.465119 & 1.220103 & 389.004 & 352.594 \\
\hline $\mathrm{H}$ & 0.267342 & 2.075080 & 1.960340 & 408.911 & 343.277 \\
\hline $\mathrm{H}$ & 1.014174 & 1.501505 & 0.715029 & 438.343 & 395.511 \\
\hline & & & & 454.005 & 419.054 \\
\hline & & & & 534.342 & 469.953 \\
\hline & & & & 569.3 & 510.973 \\
\hline & & & & 641.683 & 552.738 \\
\hline & & & & 735.916 & 658.229 \\
\hline & & & & 764.676 & 674.951 \\
\hline & & & & 809.39 & 682.591 \\
\hline & & & & 838.307 & 759.964 \\
\hline & & & & 873.898 & 764.836 \\
\hline & & & & 984.818 & 854.284 \\
\hline & & & & 1638.954 & 1610.144 \\
\hline & & & & 1651.162 & 1596.516 \\
\hline & & & & 1656.297 & 1597.282 \\
\hline & & & & 1673.822 & 1657.14 \\
\hline & & & & 1691.713 & 1591.74 \\
\hline & & & & 1702.181 & 1651.307 \\
\hline & & & & 3285.636 & 3012.266 \\
\hline & & & & 3368.044 & 3124.785 \\
\hline & & & & 3408.869 & 3249.66 \\
\hline & & & & 3501.846 & 3341.809 \\
\hline & & & & 3569.866 & 3398.507 \\
\hline & & & & 3583.246 & 3408.763 \\
\hline & & & & 3739.62 & 3549.213 \\
\hline & & & & 3880.092 & 3687.594 \\
\hline & & & & 3881.297 & 3692.73 \\
\hline & & & & 3881.709 & 3691.074 \\
\hline & & & & 3883.806 & 3691.242 \\
\hline & & & & 3886.533 & 3691.528 \\
\hline
\end{tabular}


Table S11: $\left(\mathrm{H}_{2} \mathrm{O}\right)_{6}$-Bag geometry, harmonic and VPT2 fundamental frequencies

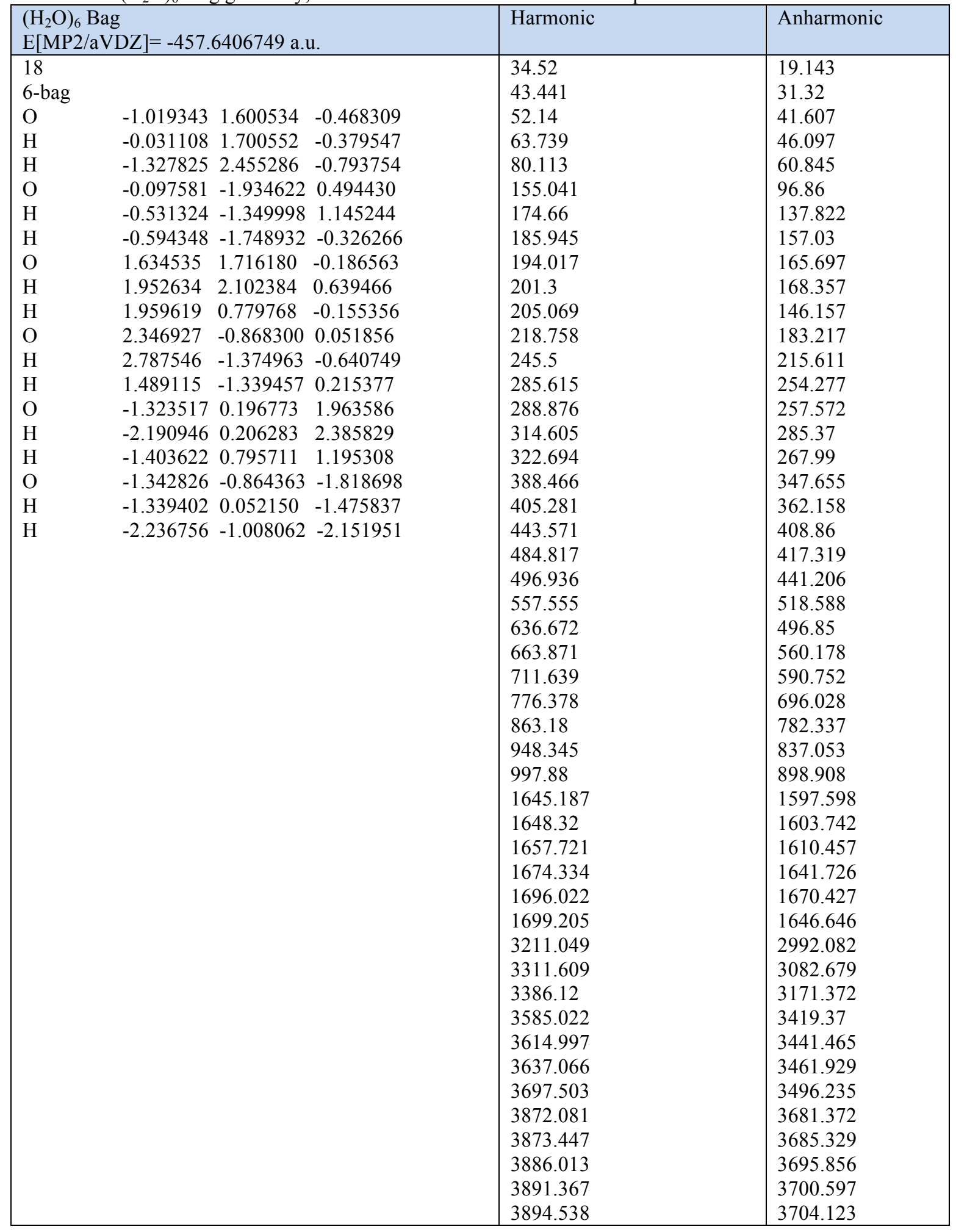


Table S12: $\left(\mathrm{H}_{2} \mathrm{O}\right)_{6}$-Cyclic boat-1 geometry, harmonic and VPT2 fundamental frequencies

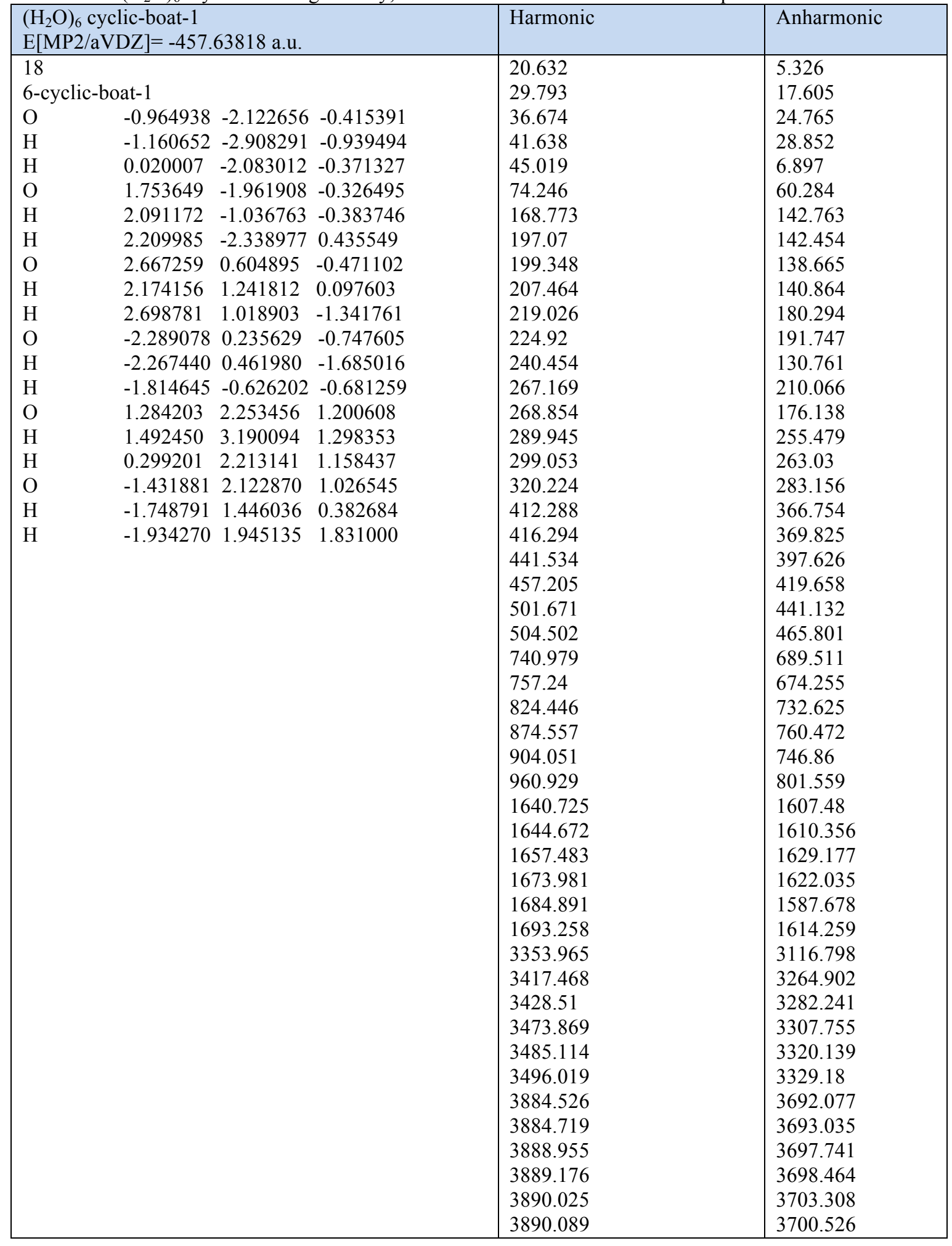


Table S13: $\left(\mathrm{H}_{2} \mathrm{O}\right)_{6}$-Cyclic boat-2 geometry, harmonic and VPT2 fundamental frequencies

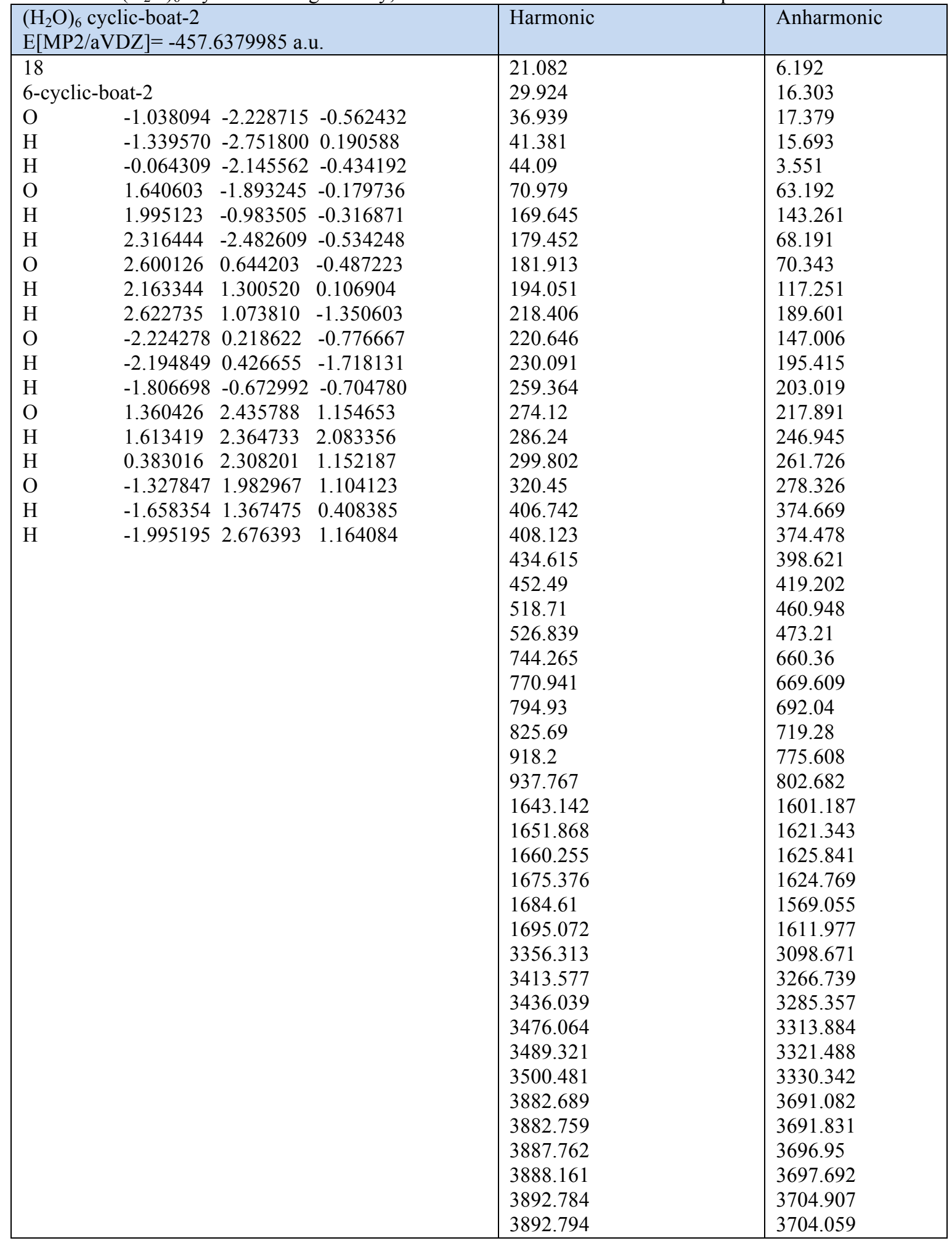


Table S14: $\left(\mathrm{H}_{2} \mathrm{O}\right)_{8}$-S4 geometry, harmonic and VPT2 fundamental frequencies

\begin{tabular}{|c|c|c|c|}
\hline \multicolumn{2}{|c|}{$\begin{array}{l}\left(\mathrm{H}_{2} \mathrm{O}\right)_{8} \mathrm{~S} 4 \\
\mathrm{E}[\mathrm{MP} 2 / \mathrm{aVDZ}]=-610.2105722 \text { a.u. }\end{array}$} & Harmonic & Anharmonic \\
\hline \multicolumn{2}{|l|}{24} & 69.203 & 54.706 \\
\hline \multirow{2}{*}{\multicolumn{2}{|c|}{ 8-S4 }} & 73.283 & 56.288 \\
\hline & & 74.274 & 63.439 \\
\hline $\mathrm{H}$ & $\begin{array}{lll}-1.348963 & -0.813 / 1 / & 1.53240 /\end{array}$ & 74.274 & 63.403 \\
\hline $\mathrm{O}$ & $\begin{array}{lll}-0.061639 & -1.900221 & 1.365265\end{array}$ & 78.806 & 65.95 \\
\hline $\mathrm{H}$ & $\begin{array}{llll}0.752336 & -1.381895 & 1.517644\end{array}$ & 108.537 & 93.55 \\
\hline $\mathrm{H}$ & $\begin{array}{lll}0.274391 & -2.704981 & -2.102680\end{array}$ & 164.848 & 141.267 \\
\hline $\mathrm{O}$ & $2.002094 \quad 0.064076 \quad 1.476004$ & 165.971 & 139.435 \\
\hline $\mathrm{O}$ & $\begin{array}{llll}-2.002081 & -0.064082 & 1.476021\end{array}$ & 165.971 & 139.45 \\
\hline $\mathrm{H}$ & $-2.704981-0.274413 \quad 2.102678$ & 174.654 & 149.77 \\
\hline & & 189.989 & 166.653 \\
\hline $\mathrm{H}$ & $\begin{array}{lll}-2.141419 & 0.008498 & -0.419807\end{array}$ & 207.401 & 182.177 \\
\hline $\mathrm{H}$ & $-0.752321 \quad 1.381885 \quad 1.517657$ & $\begin{array}{l}207.892 \\
207893\end{array}$ & $\begin{array}{l}183.401 \\
183.402\end{array}$ \\
\hline $\mathrm{O}$ & $\begin{array}{lll}0.061652 & 1.900213 & 1.365274\end{array}$ & 276.159 & $\begin{array}{l}10.402 \\
252.326\end{array}$ \\
\hline $\mathrm{H}$ & $\begin{array}{lll}0.008499 & 2.141413 & 0.419821\end{array}$ & 281.796 & 254.301 \\
\hline $\mathrm{H}$ & $\begin{array}{lll}1.348975 & 0.813710 & 1.532397\end{array}$ & 289.12 & 260.482 \\
\hline $\mathrm{O}$ & $1.900213-0.061646-1.365275$ & 292.352 & 268.364 \\
\hline $\mathrm{O}$ & $\begin{array}{lll}-1.900224 & 0.061649 & -1.365262\end{array}$ & 292.352 & 268.279 \\
\hline $\mathrm{H}$ & $-1381897-0752324-1517646$ & 305.42 & 278.815 \\
\hline $\mathrm{H}$ & - & 303.421 & 279.027 \\
\hline $\mathrm{H}$ & $\begin{array}{lll}-0.274421 & 2.704997 & -2.102653\end{array}$ & 317.162 & 292.318 \\
\hline $\mathrm{O}$ & $\begin{array}{llll}0.064067 & -2.002082 & -1.476019\end{array}$ & $\begin{array}{l}448.911 \\
454.833\end{array}$ & $\begin{array}{l}401.935 \\
404.75\end{array}$ \\
\hline $\mathrm{H}$ & $\begin{array}{lll}-0.008494 & -2.141417 & 0.419811\end{array}$ & 484.399 & 427.556 \\
\hline $\mathrm{H}$ & $\begin{array}{lll}0.813704 & -1.348967 & -1.532409\end{array}$ & 484.4 & 427.465 \\
\hline $\mathrm{H}$ & $2.141414 \quad-0.008498 \quad-0.419823$ & 505.023 & 459.581 \\
\hline $\mathrm{H}$ & $\begin{array}{lll}2.705002 & 0.274410 & 2.102651\end{array}$ & 558.832 & 493.129 \\
\hline $\mathrm{O}$ & $\begin{array}{llll}-0.064082 & 2.002090 & -1.476006\end{array}$ & $\begin{array}{l}558.832 \\
567851\end{array}$ & 493.222 \\
\hline $\mathrm{H}$ & 00127161218071 & 567.851 & 497.561 \\
\hline & $\begin{array}{lll}-0.813716 & 1.348971 & -1.532393\end{array}$ & 624.251 & 533.533 \\
\hline $\mathrm{H}$ & $\begin{array}{llll}1.381891 & 0.752331 & -1.517657\end{array}$ & $\begin{array}{l}668.652 \\
690.335\end{array}$ & $\begin{array}{l}581.487 \\
594.01\end{array}$ \\
\hline & & 690.336 & 594.062 \\
\hline & & 727.534 & 616.114 \\
\hline & & 727.534 & 616.07 \\
\hline & & 741.89 & 626.016 \\
\hline & & 827.643 & 799.891 \\
\hline & & 975.564 & 879.393 \\
\hline & & 975.565 & 879.361 \\
\hline & & 1044.677 & 943.338 \\
\hline & & 1070.102 & 965.122 \\
\hline & & 1635.952 & 1596.657 \\
\hline & & 1642.906 & 1609.015 \\
\hline & & 1642.906 & 1609.105 \\
\hline & & 1657.515 & 1623.761 \\
\hline & & 1680.517 & 1704.929 \\
\hline & & 1690.188 & 1643.809 \\
\hline & & 1690.188 & 1643.747 \\
\hline & & 1714.779 & 1680.84 \\
\hline & & 3221.746 & 2963.902 \\
\hline & & 3246.884 & 2984.411 \\
\hline & & 3275.206 & 3028.786 \\
\hline & & 3275.208 & 3028.754 \\
\hline & & 3602.329 & 3422.353 \\
\hline & & 3608.037 & 3425.331 \\
\hline & & 3608.037 & 3425.318 \\
\hline & & 3618.664 & 3431.774 \\
\hline & & 3666.032 & 3463.437 \\
\hline & & 3693.072 & 3484.688 \\
\hline & & 3693.072 & 3484.659 \\
\hline & & 3711.541 & 3497.777 \\
\hline & & 3878.265 & 3685.044 \\
\hline & & 3878.288 & 3685.47 \\
\hline & & 3878.288 & 3685.333 \\
\hline & & 3878.364 & 3685.839 \\
\hline
\end{tabular}


Table S15: $\left(\mathrm{H}_{2} \mathrm{O}\right)_{8}$-D2d geometry, harmonic and VPT2 fundamental frequencies

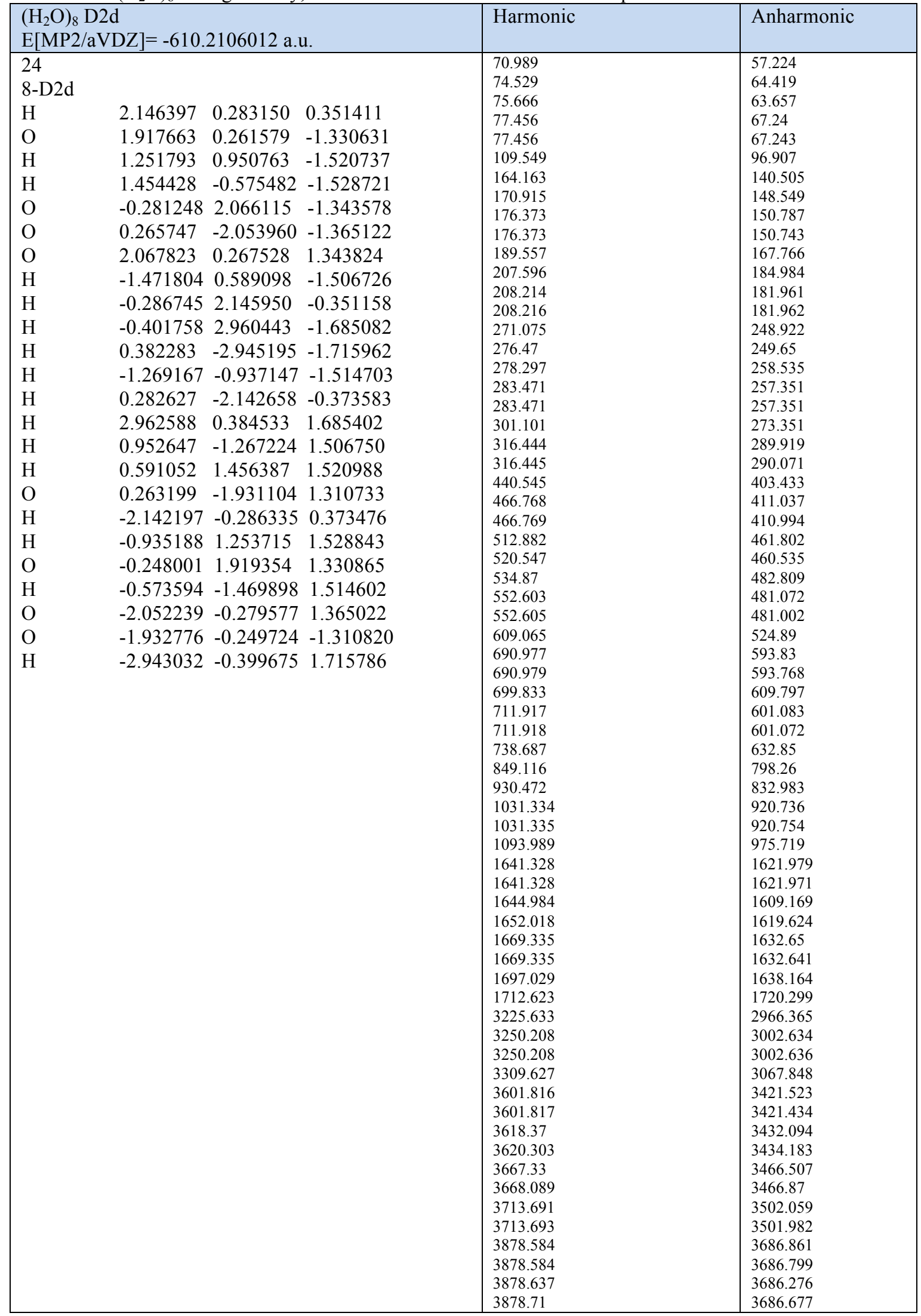


Table S16: $\left(\mathrm{H}_{2} \mathrm{O}\right)_{9}$-D2dDD geometry, harmonic and VPT2 fundamental frequencies

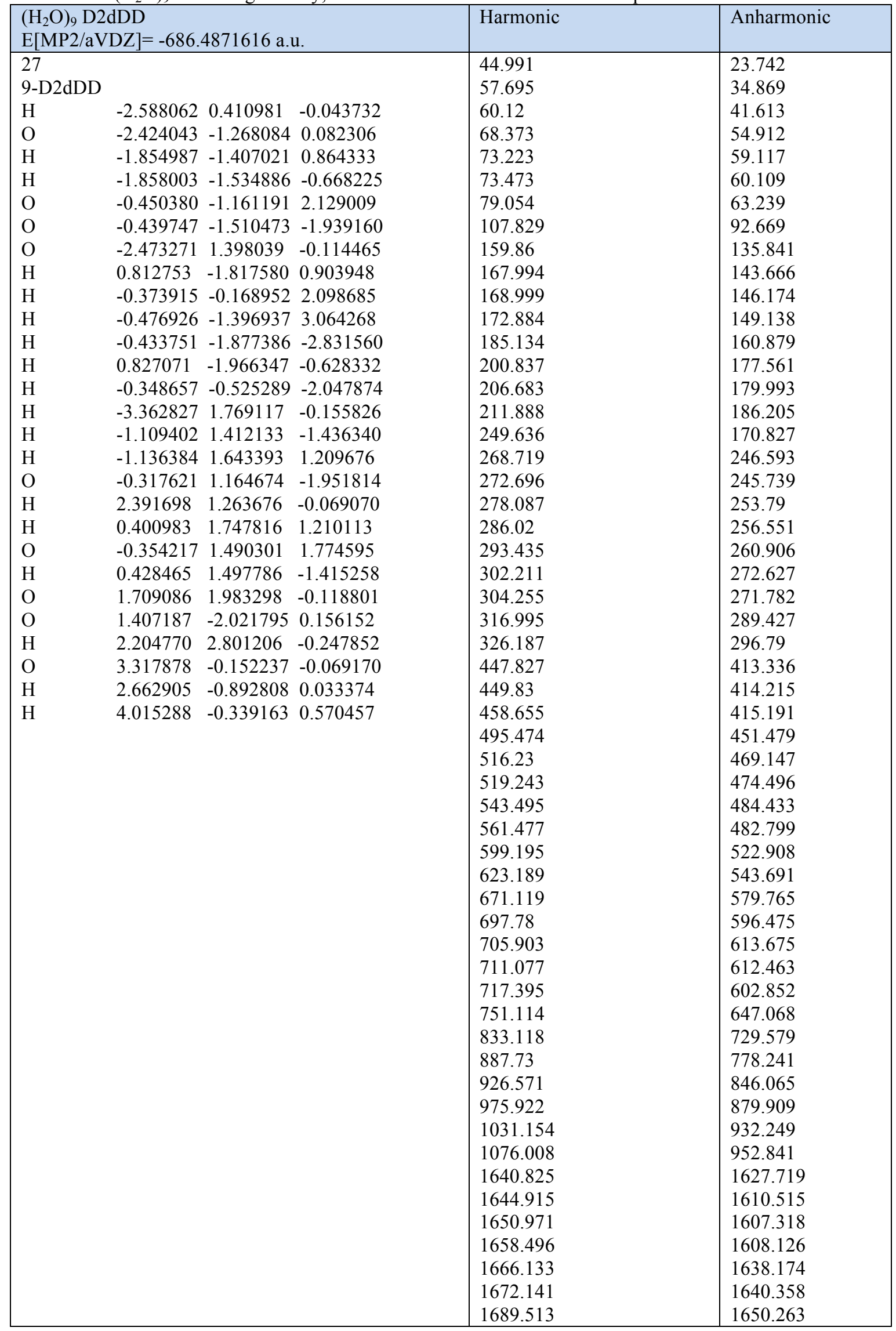




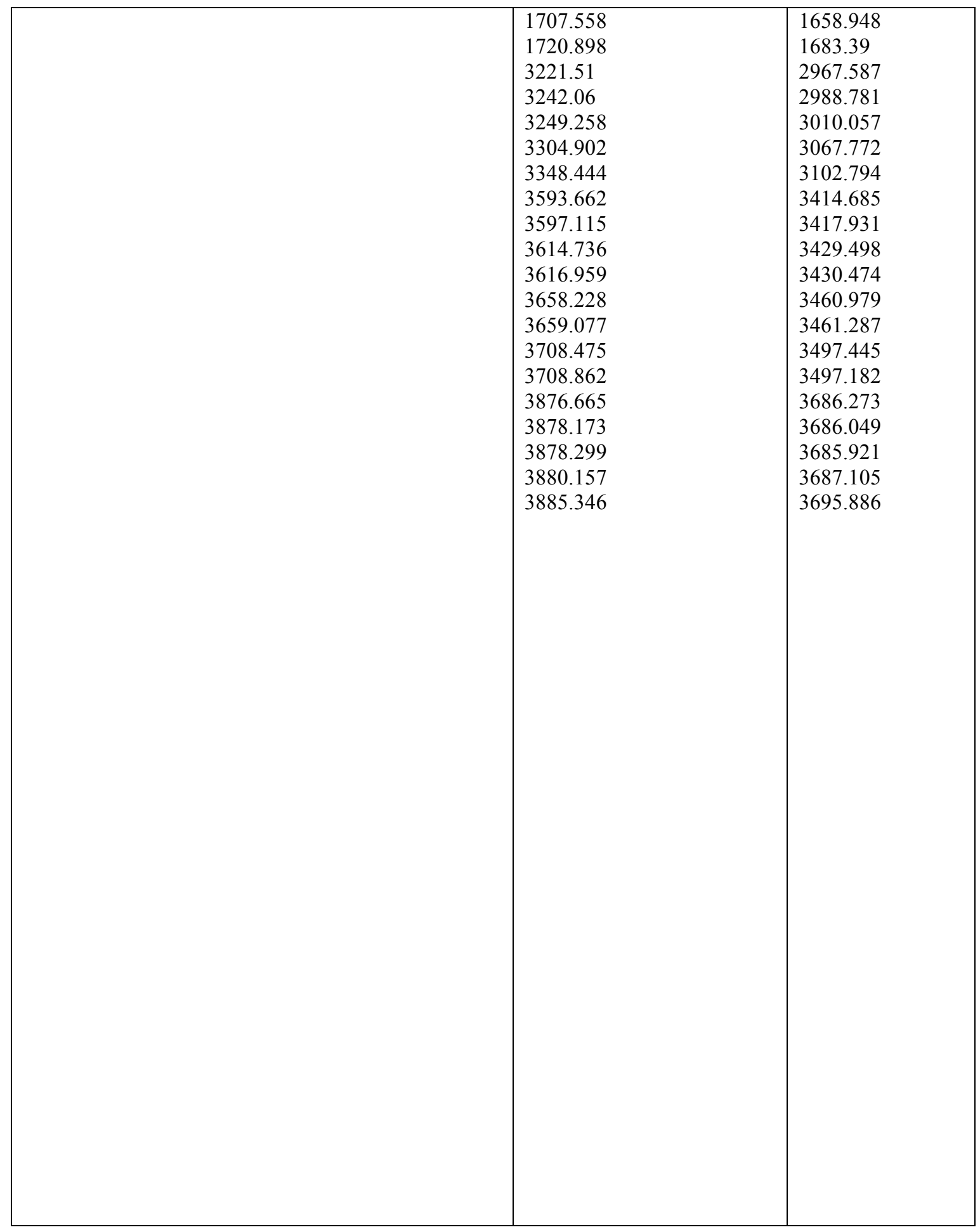


Table S17: $\mathrm{H}_{2} \mathrm{SO}_{4}\left(\mathrm{H}_{2} \mathrm{O}\right)$ geometry, harmonic and VPT2 fundamental frequencies

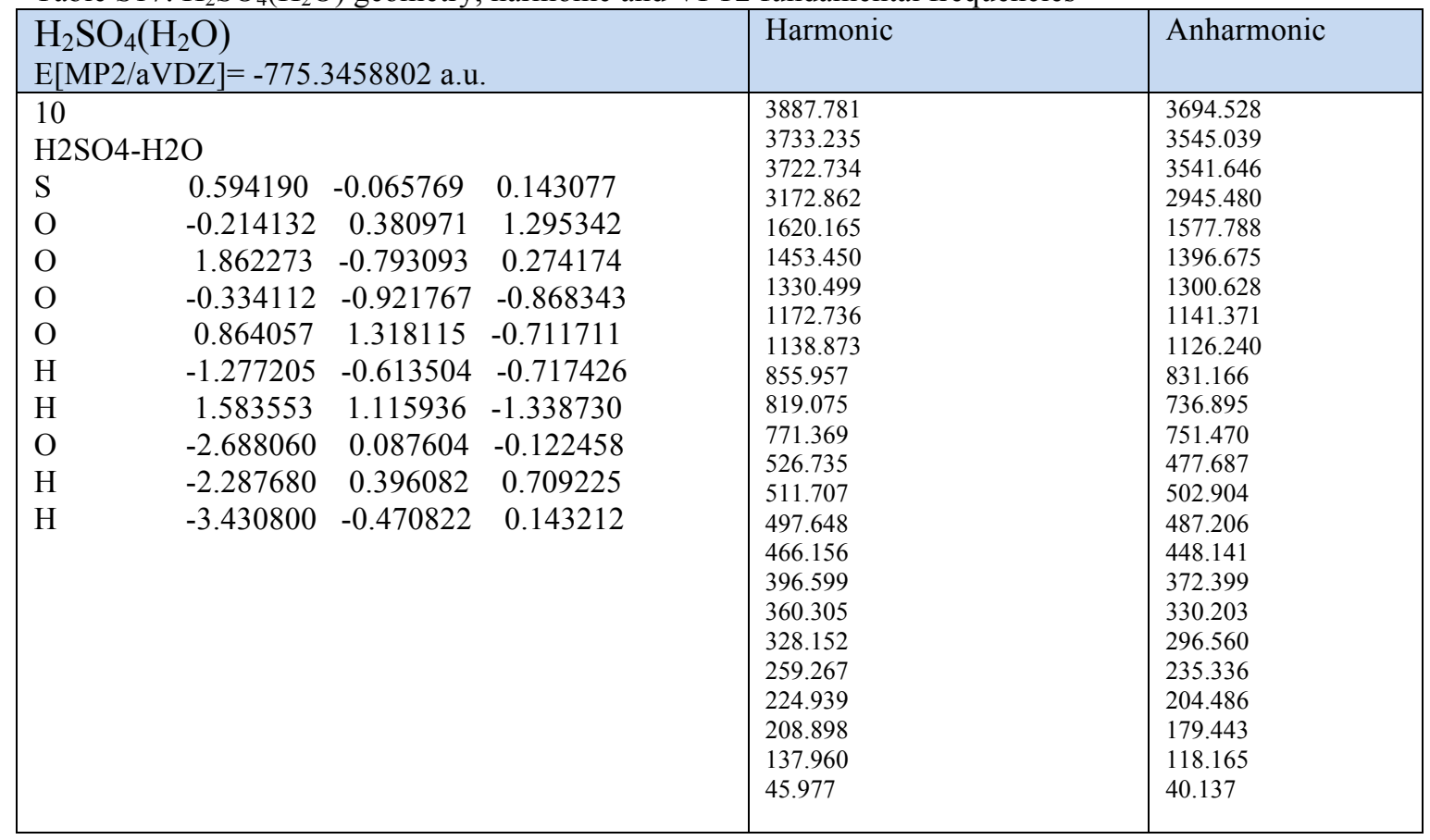

Table S18: $\mathrm{H}_{2} \mathrm{SO}_{4}\left(\mathrm{H}_{2} \mathrm{O}\right)_{2}$ geometry, harmonic and VPT2 fundamental frequencies

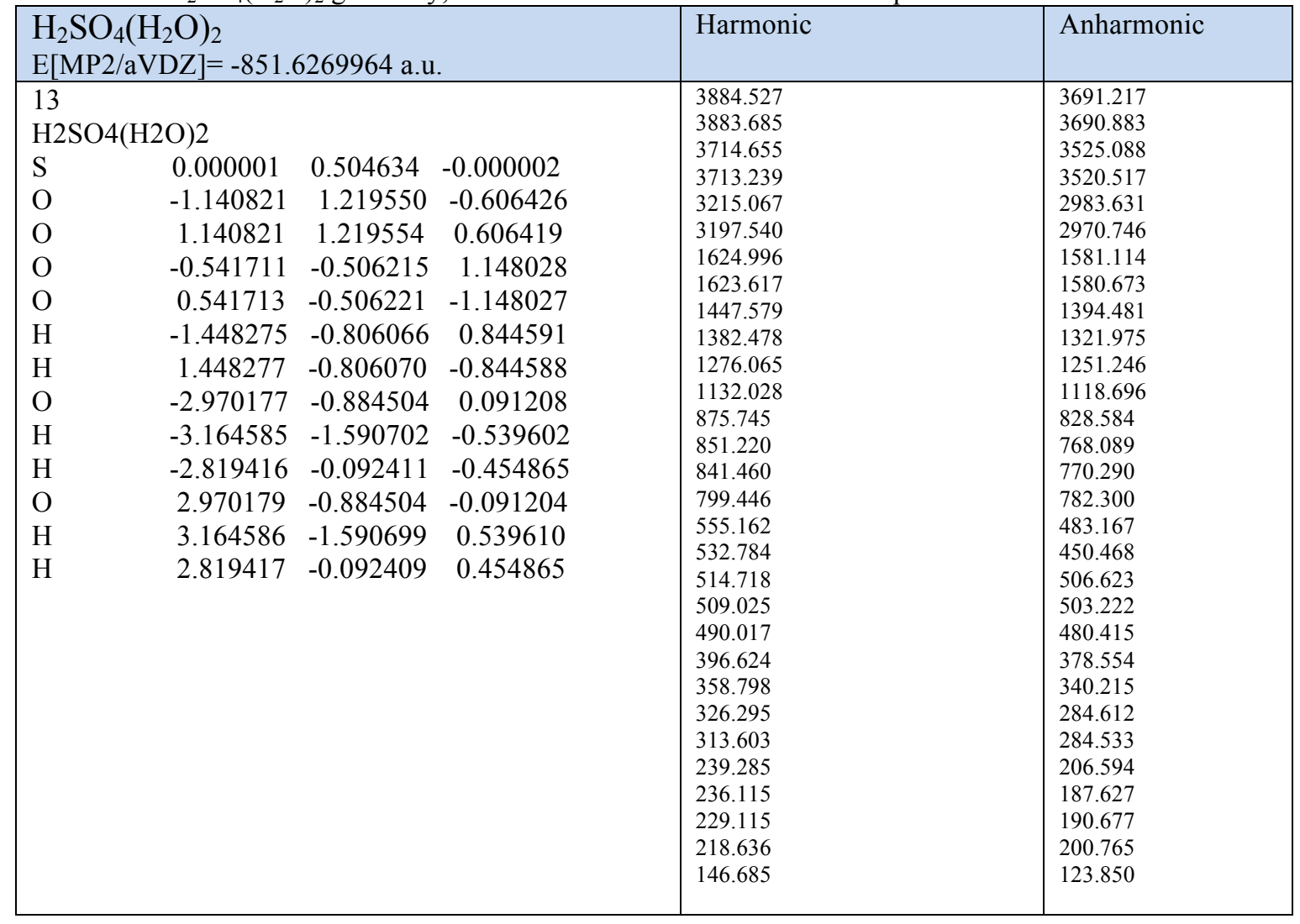

